\title{
1 Genetics of brain age suggest an overlap with common brain disorders
}

2 Tobias Kaufmann ${ }^{1, *}$, Dennis van der Meer ${ }^{1}$, Nhat Trung Doan ${ }^{1}$, Emanuel Schwarz ${ }^{2}$, Martina J.

3 Lund $^{1}$, [alphabetic order starts] Ingrid Agartz ${ }^{1,3,4}$, Dag Alnæs ${ }^{1}$, Deanna M. Barch ${ }^{5,6,7}$, Ramona

4 Baur-Streubel $^{8}$, Alessandro Bertolino ${ }^{9,10}$, Francesco Bettella ${ }^{1}$, Mona K. Beyer ${ }^{11,12}$, Erlend Bøen ${ }^{3}$,

5 Stefan Borgwardt ${ }^{13,14}$, Christine L. Brandt ${ }^{1}$, Jan Buitelaar ${ }^{15,16}$, Elisabeth G. Celius ${ }^{17,18}$, Simon

6 Cervenka ${ }^{4}$, Annette Conzelmann ${ }^{19}$, Aldo Córdova-Palomera ${ }^{1,20}$, Anders M. Dale ${ }^{21}$, Dominique J.-

7 F de Quervain ${ }^{22,23}$, Pasquale Di Carlo ${ }^{10}$, Srdjan Djurovic ${ }^{24,25}$, Erlend S. Dørum ${ }^{1,26,27}$, Sarah

8 Eisenacher $^{2}$, Torbjørn Elvsåshagen ${ }^{1,11,18}$, Thomas Espeseth ${ }^{26}$, Helena Fatouros-Bergman ${ }^{4}$, Lena

9 Flyckt $^{4}$, Barbara Franke ${ }^{28}$, Oleksandr Frei ${ }^{1}$, Beathe Haatveit ${ }^{1,26}$, Asta K. Håberg ${ }^{29,30}$, Hanne F.

10 Harbo $^{18,11}$, Catharina A. Hartman ${ }^{31}$, Dirk Heslenfeld ${ }^{32,33}$, Pieter J. Hoekstra ${ }^{34}$, Einar A.

11 Høgest $\varnothing 1^{11,18}$, Terry Jernigan ${ }^{35,36,37}$, Rune Jonassen ${ }^{26}$, Erik G. Jönsson ${ }^{1,4}$, Karolinska

12 Schizophrenia Project $(\mathrm{KaSP})^{38}$, Peter $\mathrm{Kirsch}^{39,40}$, Iwona Kłoszewska ${ }^{41}$, Knut-Kristian

13 Kolskår ${ }^{1,26,27}$, Nils Inge Landr $\varnothing^{26,3}$, Stephanie Le Hellard ${ }^{25}$, Klaus-Peter Lesch ${ }^{42,43,44}$, Simon

14 Lovestone $^{45}$, Arvid Lundervold ${ }^{46,47}$, Astri J. Lundervold ${ }^{48,49}$, Luigi A. Maglanoc ${ }^{1,26}$, Ulrik F.

15 Malt $^{11,50}$, Patrizia Mecocci ${ }^{51}$, Ingrid Melle ${ }^{1}$, Andreas Meyer-Lindenberg ${ }^{2}$, Torgeir Moberget ${ }^{1}$,

16 Linn B. Norbom ${ }^{1,26}$, Jan Egil Nordvik ${ }^{27}$, Lars Nyberg ${ }^{52}$, Jaap Oosterlaan ${ }^{32,53,54}$, Marco Papalino ${ }^{10}$,

17 Andreas Papassotiropoulos ${ }^{55,23,56}$, Paul Pauli ${ }^{8}$, Giulio Pergola ${ }^{10}$, Karin Persson ${ }^{57,58}$, Geneviève

18 Richard ${ }^{1,26,27}$, Jaroslav Rokicki ${ }^{1,26}$, Anne-Marthe Sanders ${ }^{1,26,27}$, Geir Selbæk ${ }^{58,59,17}$, Alexey A.

19 Shadrin $^{1,}$ Olav B. Smeland ${ }^{1}$, Hilkka Soininen ${ }^{60,61}$, Piotr Sowa ${ }^{12}$, Vidar M. Steen ${ }^{25,62}$, Magda

20 Tsolaki $^{63}$, Kristine M. Ulrichsen ${ }^{1,26,27}$, Bruno Vellas ${ }^{64}$, Lei Wang ${ }^{65}$, Eric Westman ${ }^{66,14}$, Georg C.

21 Ziegler $^{42}$, Mathias Zink ${ }^{2,67}$, [alphabetic order ends] Ole A. Andreassen ${ }^{1}$, Lars T. Westlye ${ }^{1,26, *}$

22 [Author byline]: for the Alzheimer's Disease Neuroimaging Initiative ${ }^{68}$, for the Pediatric Imaging,

23 Neurocognition and Genetics Study ${ }^{69}$, for the AddNeuroMed consortium ${ }^{70}$

241 NORMENT, KG Jebsen Centre for Psychosis Research, Division of Mental Health and Addiction,

25 Oslo University Hospital \& Institute of Clinical Medicine, University of Oslo, Oslo, Norway

262 Department of Psychiatry and Psychotherapy, Central Institute of Mental Health, Medical Faculty

27 Mannheim, Heidelberg University, Mannheim, Germany

283 Department of Psychiatry, Diakonhjemmet Hospital, Oslo, Norway

294 Department of Clinical Neuroscience, Center for Psychiatry Research, Karolinska Institutet and

30 Stockholm County Council, Stockholm, Sweden

315 Department of Psychological and Brain Sciences, Washington University in St. Louis, St. Louis, USA

326 Department of Psychiatry, Washington University in St. Louis, St. Louis, USA 
Kaufmann et al., Genetics of brain age suggest an overlap with common brain disorders

7 Department of Radiology, Washington University in St. Louis, St. Louis, USA

8 Department of Psychology I, University of Würzburg, Würzburg, Germany

9 Institute of Psychiatry, Bari University Hospital, Bari, Italy

10 Department of Basic Medical Science, Neuroscience, and Sense Organs, University of Bari, Bari, Italy.

11 Institute of Clinical Medicine, University of Oslo, Oslo, Norway

12 Department of Radiology and Nuclear Medicine, Section of Neuroradiology, Oslo University Hospital,

\section{Oslo, Norway}

13 Department of Psychiatry (UPK), University of Basel, Basel, Switzerland

\section{Institute of Psychiatry, King's College, London, UK}

15 Department of Cognitive Neuroscience, Donders Institute for Brain, Cognition and Behaviour,

Radboud University Medical Center, Nijmegen, The Netherlands

16 Karakter Child and Adolescent Psychiatry University Centre, Nijmegen, The Netherlands

17 Institute of Health and Society, University of Oslo, Oslo, Norway

18 Department of Neurology, Oslo University Hospital, Oslo, Norway

19 Children and Adolescence Psychiatry, University of Tübingen, Tübingen, Germany

20 Department of Pediatrics, Stanford University School of Medicine, Stanford University, Stanford, USA

21 Department of Radiology, University of California, San Diego, La Jolla, CA, USA; Department of Neurosciences, University of California, San Diego, La Jolla, CA, USA

22 Division of Cognitive Neuroscience, University of Basel, Basel, Switzerland

23 Transfaculty Research Platform Molecular and Cognitive Neurosciences, University of Basel, Basel,

\section{Switzerland}

24 Department of Medical Genetics, Oslo University Hospital, Oslo, Norway

25 NORMENT, K.G. Jebsen Center for Psychosis Research, Department of Clinical Science, University of Bergen, Bergen, Norway

26 Department of Psychology, University of Oslo, Oslo, Norway

27 Sunnaas Rehabilitation Hospital HT, Nesodden, Norway

28 Departments of Human Genetics and Psychiatry, Donders Institute for Brain, Cognition and

Behaviour, Radboud University Medical Center, Nijmegen, The Netherlands

29 Department of Neuromedicine and Movement Science, Norwegian University of Science and Technology, Trondheim, Norway The Netherlands 
Kaufmann et al., Genetics of brain age suggest an overlap with common brain disorders

34 Department of Child and Adolescent Psychiatry, University Medical Center Groningen, University of Groningen, Groningen, The Netherlands

35 Center for Human Development, University of California, San Diego, USA

36 Department of Cognitive Science, University of California, San Diego, USA

37 Departments of Psychiatry and Radiology, University of California, San Diego, USA

38 Members of Karolinska Schizophrenia Project (KaSP) are listed before References

39 Department of Clinical Psychology, Central Institute of Mental Health, Medical Faculty Mannheim,

Heidelberg University, Mannheim, Germany

40 Bernstein Center for Computational Neuroscience Heidelberg/Mannheim, Mannheim, Germany

41 Department of Old Age Psychiatry and Psychotic Disorders, Medical University of Lodz, Poland

42 Division of Molecular Psychiatry, Center of Mental Health, University of Würzburg, Würzburg,

Germany

43 Laboratory of Psychiatric Neurobiology, Institute of Molecular Medicine, Sechenov First Moscow

State Medical University, Moscow, Russia

44 Department of Neuroscience, School for Mental Health and Neuroscience (MHeNS), Maastricht

University, Maastricht, The Netherlands

45 Department of Psychiatry, Warneford Hospital, University of Oxford, Oxford, UK

46 Department of Biomedicine, University of Bergen, Norway

47 Mohn Medical Imaging and Visualization Centre, Department of Radiology, Haukeland University

Hospital, Bergen, Norway

48 Department of Biological and Medical Psychology, University of Bergen, Norway

49 K. G. Jebsen Centre for Neuropsychiatric Disorders, University of Bergen, Norway

50 Department of Research and Education, Oslo University Hospital, Oslo, Norway

51 Institute of Gerontology and Geriatrics, University of Perugia, Perugia, Italy

9252 Department of Radiation Science, Umeå University, Umeå, Sweden

9353 Emma Children's Hospital Amsterdam Medical Center, Amsterdam, The Netherlands

9454 VU University Medical Center, Department of Pediatrics, Amsterdam, The Netherlands

9555 Division of Molecular Neuroscience, University of Basel, Basel, Switzerland

9656 Life Sciences Training Facility, Department Biozentrum, University of Basel, Basel, Switzerland

9757 Department of Geriatric Medicine, Oslo University Hospital, Oslo, Norway

9858 Norwegian National Advisory Unit on Ageing and Health, Vestfold Hospital Trust, Tønsberg, Norway

9959 Centre for Old Age Psychiatric Research, Innlandet Hospital Trust, Ottestad, Norway

10060 Department of Neurology, Institute of Clinical Medicine, University of Eastern Finland, Kuopio,

$101 \quad$ Finland

10261 Neurocenter, Neurology, Kuopio University Hospital, Kuopio, Finland 
Kaufmann et al., Genetics of brain age suggest an overlap with common brain disorders

62 Dr. E. Martens Research Group for Biological Psychiatry, Department of Medical Genetics, Haukeland University Hospital, Bergen, Norway.

$631^{\text {st }}$ Department of Neurology, Aristotle University of Thessaloniki, Makedonia, Greece. 64 INSERM U 1027, University of Toulouse, Toulouse, France 66 Department of Neurobiology, Care Sciences and Society, Karolinska Institute, Stockholm, Sweden 67 District hospital Ansbach, Germany 68 Data used in preparation of this article were obtained from the Alzheimer's Disease Neuroimaging Initiative (ADNI) database (adni.loni.usc.edu). As such, the investigators within the ADNI contributed to the design and implementation of ADNI and/or provided data but did not participate in analysis or writing of this report. A complete listing of ADNI investigators can be found at: http://adni.loni.usc.edu/wp-content/uploads/how_to_apply/ADNI_Acknowledgement_List.pdf.

69 Data used in preparation of this article were obtained from the Pediatric Imaging, Neurocognition and Genetics Study (PING) database (http://ping.chd.ucsd.edu). As such, the investigators within PING contributed to the design and implementation of PING and/or provided data but did not participate in

124 Tobias Kaufmann, Ph.D. \& Lars T. Westlye, Ph.D.

125 Email: tobias.kaufmann@medisin.uio.no, 1.t.westlye@psykologi.uio.no

126 Postal address: OUS, PoBox 4956 Nydalen, 0424 Oslo, Norway

127 Telephone: +47230273 50, Fax: +4723027333

\section{Counts:}

130 Abstract: 144 words

131 Main text body: 3147 words

132 References: 41 in the main paper, 111 incl. supplemental

133 Figures: 5 (1x 1-column, 4x 2-column)

135 Key words: Brain age gap, brain disorders, genetic architecture, pleiotropy 
Kaufmann et al., Genetics of brain age suggest an overlap with common brain disorders

136 Numerous genetic and environmental factors contribute to psychiatric disorders and other

137 brain disorders. Common risk factors likely converge on biological pathways regulating the

138 optimization of brain structure and function across the lifespan. Here, using structural

139 magnetic resonance imaging and machine learning, we estimated the gap between brain age

140 and chronological age in 36,891 individuals aged 3 to 96 years, including individuals with

141 different brain disorders. We show that several disorders are associated with accentuated

142 brain aging, with strongest effects in schizophrenia, multiple sclerosis and dementia, and

143 document differential regional patterns of brain age gaps between disorders. In 16,269

144 healthy adult individuals, we show that brain age gap is heritable with a polygenic

145 architecture overlapping those observed in common brain disorders. Our results identify

146 brain age gap as a genetically modulated trait that offers a window into shared and distinct

147 mechanisms in different brain disorders.

148 Psychiatric disorders and other brain disorders are among the main contributors to morbidity and

149 disability around the world ${ }^{1}$, often placing debilitating disadvantages on the individual ${ }^{2}$. The

150 disease mechanisms are complex, spanning a wide range of genetic and environmental

151 contributing factors ${ }^{3}$. The inter-individual variability is large, but on a group-level, patients with

152 common brain disorders perform worse on cognitive tests, are less likely to excel professionally,

153 and engage in adverse health behaviours more frequently ${ }^{4}$.

154 Dynamic processes influencing the rate of maturation and change throughout the lifespan

155 play a critical role, as reflected in the wide range of disease onset times from early childhood to

156 old age ${ }^{5}$. This suggests that the age at which individual trajectories diverge from the norm is a

157 key characteristic in the underlying pathophysiology. Whereas autism spectrum disorder (ASD)

158 and attention-deficit/hyperactivity disorder (ADHD) have onset in childhood ${ }^{6}$, schizophrenia 
Kaufmann et al., Genetics of brain age suggest an overlap with common brain disorders

159 (SZ) and bipolar (BD) spectrum disorders likely evolve during adolescence, before the outbreak

160 of severe symptoms, which is typically in early adulthood ${ }^{6,7}$. Likewise, multiple sclerosis (MS)

161 most often presents itself in early adulthood but the disease process likely starts much earlier ${ }^{8,9}$.

162 First episodes in major depressive disorder (MDD) can appear at any stage from adolescence to

163 old age $\mathrm{e}^{6,10}$, whereas mild cognitive impairment (MCI) and dementia (DEM) most often evolve in

164 old age $\mathrm{e}^{11}$. When attempting to decode the underlying brain dysfunction of these disorders, age-

165 related deviations from the norm may also differ in terms of spatial location, direction, change

166 rate and magnitude, all of which add complexity to the interpretation of observed effects.

167 Magnetic resonance imaging (MRI) is a powerful tool to unveil abnormal brain 168 development ${ }^{12,13}$ and age-related degeneration ${ }^{14,15}$. Machine learning techniques enable robust 169 estimation of the biological age of the brain using MRI-derived features ${ }^{16}$, and initial evidence 170 suggests that a deviation between brain and chronological age - termed the brain age gap - is 171 present in several brain disorders ${ }^{17}$. These findings may render brain age gap a promising marker 172 of brain health ${ }^{17}$, but several critical issues remain to be addressed. First, while advantageous for 173 narrowing the complexity, reducing a rich set of brain imaging features into a single estimate of 174 brain age inevitably compromises spatial specificity, thereby potentially removing disorder175 specific patterns. Second, most studies so far have been rather small-scale, performed within a 176 limited age range and focusing on a single disorder, which left them unable to uncover clinical 177 specificity and lifespan dynamics. Third, the genetic underpinnings of brain age gap are not 178 understood and it is unknown to what degree they overlap with the genetic architecture of major 179 clinical traits. To address these critical knowledge gaps, large imaging genetics samples covering 180 a range of prevalent brain disorders are necessary.

The availability of unprecedented sample sizes of neuroimaging and genetics data through 182 global data sharing and population-based efforts provide new opportunities for accurate 
Kaufmann et al., Genetics of brain age suggest an overlap with common brain disorders

183 modelling of lifespan differences in brain anatomy and its application to brain disorders ${ }^{18}$. Here,

184 we have gathered raw structural MRI data from a large number of individuals. We employed a

185 centralized and harmonized processing protocol including automated surface-based morphometry

186 and subcortical segmentation using Freesurfer ${ }^{19}$ (Suppl. Fig. 1). The main analysis in this study

187 is based on data from 36,891 individuals aged 3 to 96 years that passed quality control,

188 representing the largest brain imaging study on brain age to date.

189 This sample included data from healthy controls (HC; $n=30,967 ; 3-95$ years), as well as

190 from 5924 individuals with diverse brain disorders with typical onset age distributed across the

191 lifespan ${ }^{6,11}$. We included data from individuals with ASD $(n=975 ; 5-64$ years $)$ and ADHD $(n=$

$192751 ; 7-62$ years), individuals with prodromal SZ or at risk mental state (SZRISK; $n=98 ; 16-42$

193 years), individuals with SZ ( $\mathrm{n}=1,145 ; 18-66$ years), a heterogeneous group with mixed

194 diagnoses in the psychosis spectrum (PSYMIX; $n=294 ; 18-69$ years), individuals with BD ( $n=$

$195445 ; 18-66$ years $),$ MS $(n=254 ; 19-68$ years $), \operatorname{MDD}(n=211 ; 18-71$ years $)$, MCI $(n=992 ; 38-91$

196 years), and DEM (including Alzheimer's disease (AD); $n=759 ; 53-96$ years). Supplementary

197 Tables 1 and 2 provide details on the sample's characteristics.

198 Brain age prediction across brain disorders

199 We used machine learning to estimate individual biological brain age based on structural brain

200 imaging features. First, we grouped all subjects into different samples. For each of the ten clinical

201 groups, we identified a group of healthy individuals of equal size, matched on age, sex and

202 scanning site using propensity score matching ${ }^{20}$. All remaining individuals were joined into one

203 sample comprising healthy individuals only. The latter constituted a training sample, used to train

204 and tune the machine learning models for age prediction $(n=26,535 ; 14,182$ females and 12,353 
Kaufmann et al., Genetics of brain age suggest an overlap with common brain disorders

males, aged 3-89 years), whereas the ten clinical samples were used as independent test samples.

206 Figure 1a illustrates the respective age distributions per sex and diagnosis.

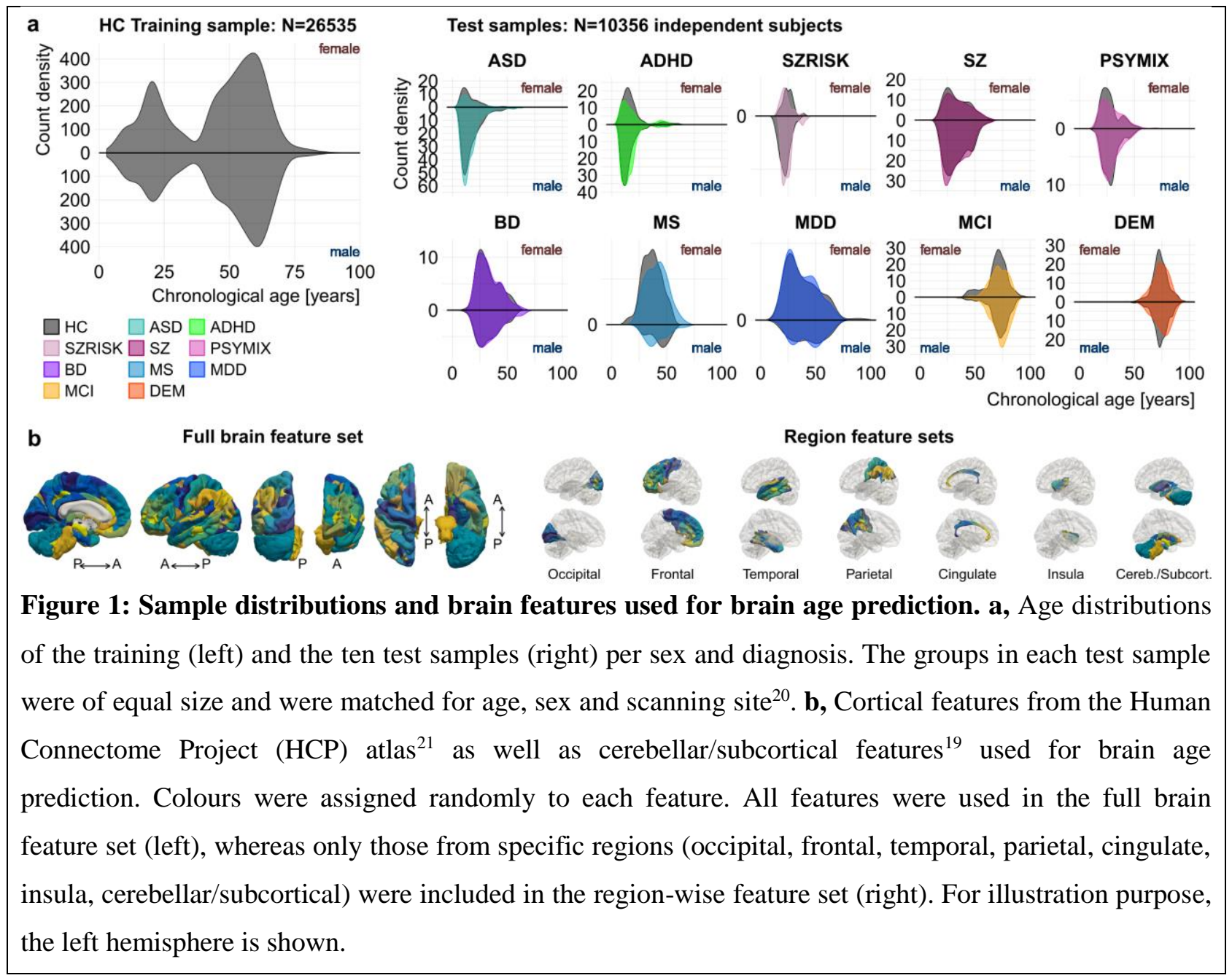

The large sample size and wide age-span of the training sample allowed us to model male

208 and female brain age separately, thereby accounting for potential sexual dimorphisms in brain

209 structural lifespan trajectories. For each sex, we built a machine learning model based on gradient

210 tree boosting (xgboost $)^{22}$ to predict the age of the brain from a set of thickness, area and volume

211 features extracted using a multi-modal parcellation of the cerebral cortex ${ }^{21}$ as well as a set of

212 cerebellar/subcortical volume features ${ }^{19}$ (1,118 features in total, Fig. 1b). Five-fold cross-

213 validations confirmed the validity of the models, yielding high correlations between

214 chronological age and predicted brain age $(r=.94$ and $r=.95$ for the female and male model, 
Kaufmann et al., Genetics of brain age suggest an overlap with common brain disorders

215 respectively; Suppl. Fig. 2). Next, we applied the models to predict brain age for each individual

216 in the ten independent test samples, and tested for effects of diagnosis on the brain age gap. We

217 used mega-analysis (across-site analysis) as the main statistical framework as it may best exploit

218 the benefits of the big data approach, while also providing results from a meta-analysis

219 framework in the supplement. We controlled all associations and group differences reported in

220 this paper for age, age ${ }^{2}$, sex, and scanning site. Further, to rule out confounding effects of data

221 quality on the results ${ }^{23}$, we repeated the main analyses using a more stringent multivariate quality

222 control and exclusion procedure ${ }^{24}$.

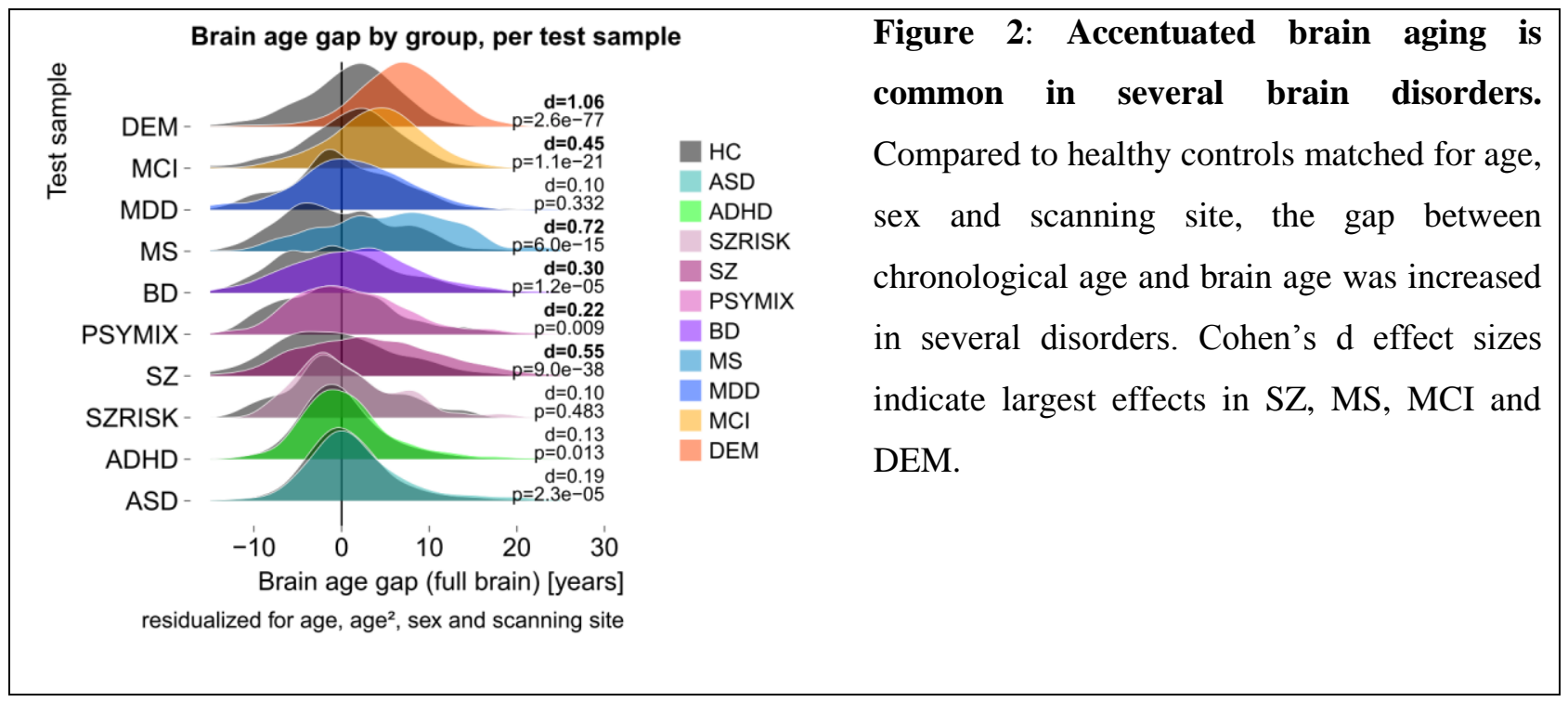

Figure 2 illustrates that the brain age gap was increased in several brain disorders.

224 Strongest effects were observed in SZ (Cohen's $d=0.55)$, MS $(d=0.72)$, MCI $(d=0.45)$ and

$225 \operatorname{DEM}(d=1.06)$. PSYMIX $(d=0.22)$ and BD $(d=0.30)$ showed small effects of increased brain

226 age gap, whereas other groups showed negligible effects. The meta-analysis converged on the

227 same findings (Suppl. Fig. 3) and the results replicated regardless of the quality control exclusion

228 criterion applied (Suppl. Fig. 4). Compared to matched healthy controls, the average brain age

229 gap was estimated to 1.1 years for ASD, 0.7 years for ADHD, 0.6 years for SZRISK, 3.9 years

230 for SZ, 1.4 years for PSYMIX, 2.0 years for BD, 5.6 years for MS, 0.8 years for MDD, 3.0 years 
Kaufmann et al., Genetics of brain age suggest an overlap with common brain disorders

231 for MCI and 5.8 years for DEM. The brain age gap in all clinical groups was positive and there

232 were no signs of a negative brain age gap (delay) in children with ASD or ADHD.

\section{Regional specificity of brain age gap}

We assessed the specificity of the spatial brain age gap patterns across clinical groups. We

trained age prediction models similar to those for the full brain above, including only occipital,

236 frontal, temporal, parietal, cingulate, insula, or cerebellar/subcortical features (Fig. 1b). Cross-

237 validation confirmed the predictive performance of all regional models (Suppl. Fig. 2), so we

238 used these to predict regional brain age in the ten independent test sets. Region-wise brain age

239 gaps often corresponded to the ones observed on the full brain level, yet some notable differences

240 in the spatial patterns of the disorders emerged (Fig. 3a). For example, increased

241 cerebellar/subcortical age gap was most prominent in DEM $(d=0.99)$ and MS $(d=0.89)$ but was

242 not present in SZ $(d=0.08)$. The largest effect in SZ was observed in the frontal lobe $(d=0.71)$.

243 In PSYMIX, brain age gaps in the insula $(d=0.39)$ and the temporal lobe $(d=0.34)$ were larger

244 than the brain age gap observed on the full brain level $(d=0.22)$. A brain age gap in the temporal

245 lobe was observed in MDD $(d=0.26)$, whereas there was no evidence for a brain age gap in ASD

246 or ADHD in any of the regions.

Figure 3b illustrates a hierarchical clustering of clinical groups based on region-wise

248 effect sizes. One cluster of groups with similar spatial brain age gap patterns comprised MS, MCI

249 and DEM, whereas the other groups formed a second cluster. Notably, the spatial patterns of the

250 groups in the first cluster were negatively associated with several disorders in the second cluster,

251 pointing toward spatial specificity of these disorders. 
Kaufmann et al., Genetics of brain age suggest an overlap with common brain disorders

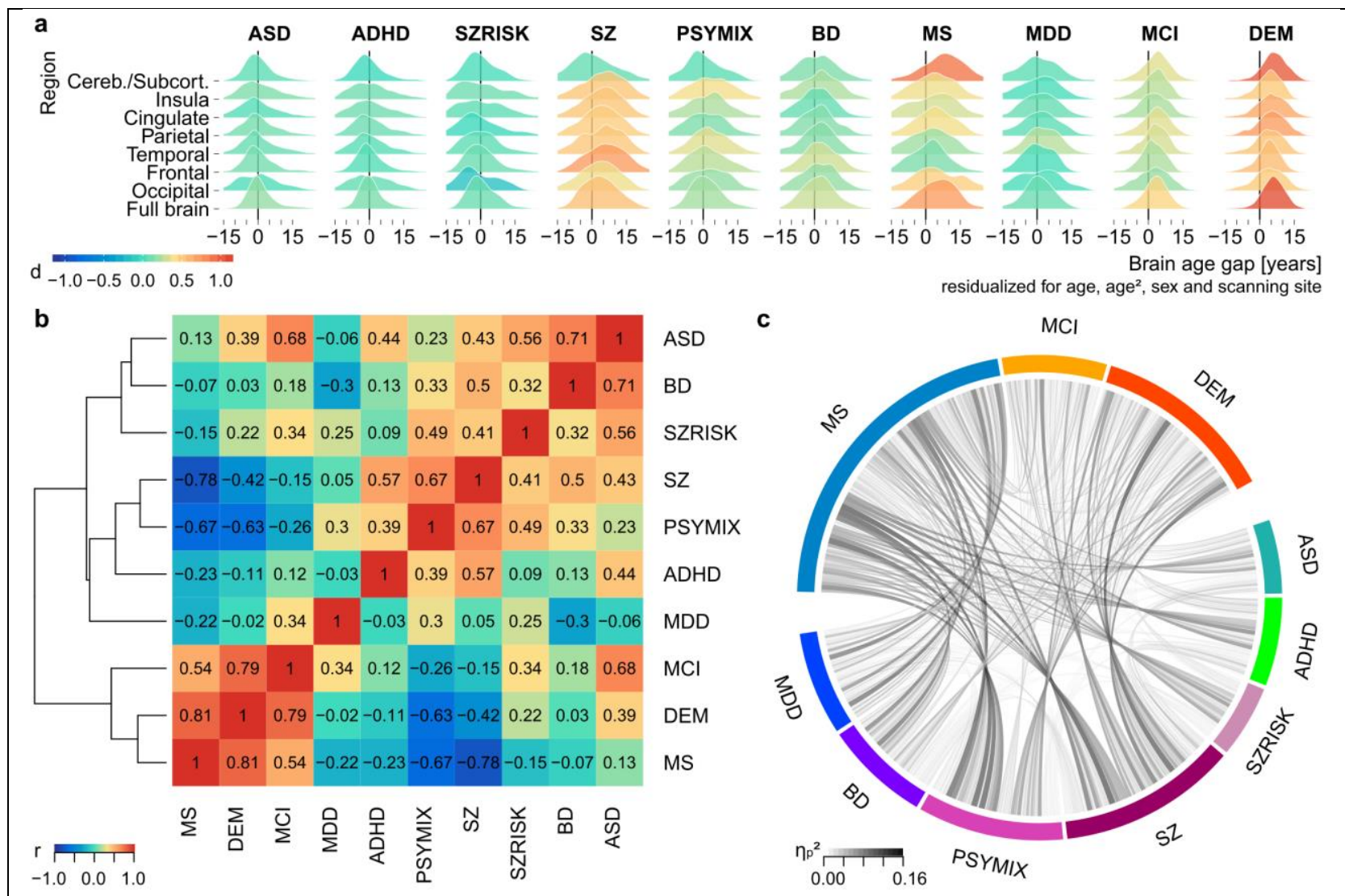

Figure 3: Several disorders displayed regional specific aging patterns. a, Region-wise brain age gaps per disorder. Colours indicate Cohen's d effect sizes for group comparison to healthy controls matched for age, sex and scanning site. Strongest patterns were observed in the cerebellar/subcortical region for DEM and MS, and in the frontal lobe for SZ. b, Correlation matrix based on the effect sizes from panel (a) indicates similarities (e.g. between MS, DEM and MCI) and dissimilarities (e.g. between SZ/PSYMIX and MS/DEM) in the spatial brain age patterns between the disorders. Sorting is based on hierarchical clustering. c, Effect sizes of region x group interaction effects from repeated measures ANOVAs run for each combination of regions and groups (1260 tests in total). Strongest interaction effects were observed between MS/MCI/DEM and the other disorders, confirming the dissimilarity pattern from panel (b) with an independent analysis.

To explore these differences further, we tested for group $\mathrm{x}$ region interactions on each

253 pairwise combination of clinical groups and pairwise combination of region-wise brain age gaps

254 (1260 tests). Figure 3c illustrates the effect sizes for all resulting group x region interactions.

255 Confirming the results from Figure 3b, strongest interaction effects were observed between the 256 groups from cluster 1 and those from cluster 2. For example, the three strongest interaction 
Kaufmann et al., Genetics of brain age suggest an overlap with common brain disorders

257 effects indicated that brain age gaps for frontal and cerebellar/subcortical regions diverged mostly

258 between MS and SZ (Partial Eta squared $\left.\eta_{p}^{2}=0.16\right)$, MS and PSYMIX $\left(\eta_{p}^{2}=0.15\right)$ and between

259 SZ and DEM ( $\eta_{p}{ }^{2}=0.15$; Suppl. Fig. 5 provides effect sizes for all tests). Together, these results

260 demonstrate that several common disorders affecting the brain show anatomically differential

261 patterns of increased brain age gap, indicating that the rate at which different regions age in

262 relation to each other oftentimes showed opposite patterns between disorders typically considered

263 neurodevelopmental and neurodegenerative disorders, respectively.

264 With converging findings suggesting largest brain age gaps in SZ, MS, MCI and DEM,

265 we explored the functional relevance of the region-wise brain age gaps for these groups, testing

266 for associations with clinical and cognitive data. Clinical data available in the SZ test sample

267 included symptom $(n=81 \mathrm{HC}, n=391 \mathrm{SZ})$ and function $(n=271 \mathrm{SZ})$ scores of the Global

268 Assessment of Functioning scale ${ }^{25}(\mathrm{GAF})$ as well as positive $(n=57 \mathrm{HC}, n=653 \mathrm{SZ})$ and

269 negative ( $n=57 \mathrm{HC}, n=655 \mathrm{SZ}$ ) scores of the Positive and Negative Syndrome Scale ${ }^{26}$

270 (PANSS). In the MS test sample, we assessed associations with scores from the Expanded

271 Disability Status Scale ${ }^{27}$ (EDSS, $n=188 \mathrm{MS}$ ) and in the joint MCI and DEM test samples, we

272 assessed associations with Mini Mental State Examination scores ${ }^{28}$ (MMSE, $n=901 \mathrm{HC}, n=921$

273 MCI, $n=707$ DEM). Figure 4a depicts association strengths for all tests and Figure 4b

274 illustrates the strongest association for each test, except for the PANSS scores where only weak

275 associations were found. In SZ, larger brain age gaps were associated with lower functioning, in

276 particular for full brain, frontal, temporal and insula brain age gaps (GAF function all $\mathrm{z}<-0.18$,

277 all $P<0.003$; GAF symptom all $z<-0.20$, all $P<2 \times 10^{-5}$ ). In MS, larger brain age gap was

278 associated with higher disability, in particular for the full brain age gap $(z=0.23, P=.001)$.

279 Finally, lower cognitive functioning was associated with larger brain age gaps in the joint 
Kaufmann et al., Genetics of brain age suggest an overlap with common brain disorders

MCI/DEM samples, with strongest effects for full brain $\left(z=-0.34, P=4 \times 10^{-65}\right)$ and

cerebellar/subcortical $\left(\mathrm{z}=-0.31, P=2 \times 10^{-53}\right)$ brain age gaps.

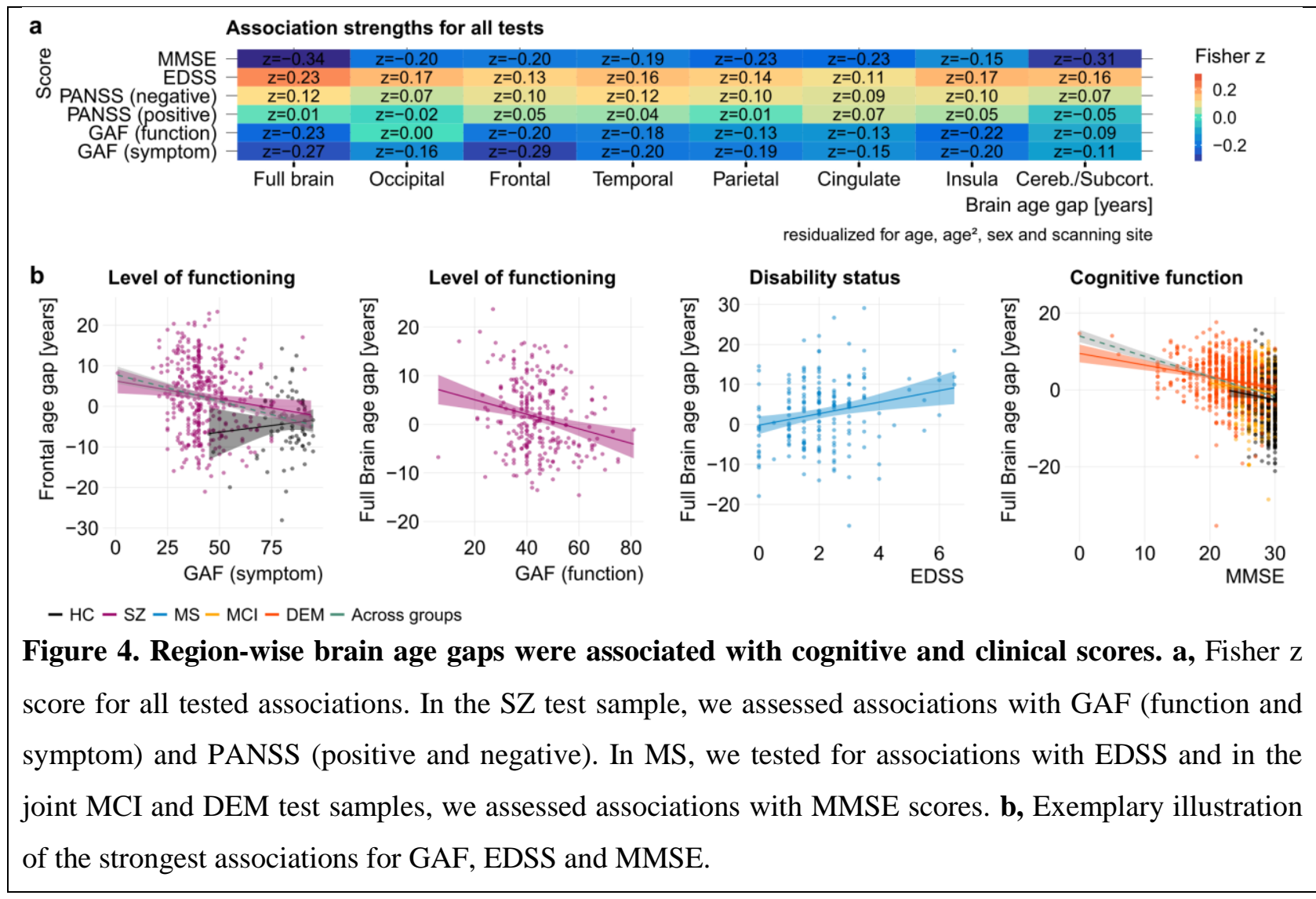

\section{The genetic architecture of brain age gap}

283 Given the known genetic contributions to brain disorders, our results pose the question to what

284 degree brain age patterns are genetically constrained and if the implied genes overlap with the

285 polygenic architectures of the disorders. In our cohorts, single nucleotide polymorphism (SNP)

286 data were available for 16,269 adult healthy controls with European ancestry, after disregarding

287 subsets with data from clinical groups, children and individuals with non-European ancestry, all

288 of which were too small to warrant an analysis. We estimated full and region-wise brain age for

289 these individuals using 5-fold cross-validation in a model trained on all healthy controls $(n=$

$29030,967)$ and regressed age, age ${ }^{2}$, sex, and scanning site effects from the resulting brain age gaps. 
Kaufmann et al., Genetics of brain age suggest an overlap with common brain disorders

First, we ran genome-wide complex trait analysis $\left(\mathrm{GCTA}^{29}\right)$ across all 16,269 individuals,

292 including the first four population components from multidimensional scaling as covariates to

293 further control for population stratification. The results revealed significant heritability (Fig. 5a),

294 with common SNPs explaining $18.3 \%$ of the variance in brain age gap across all individuals (full

295 brain, $\left.\mathrm{h}^{2} \mathrm{SNP}=0.1828, \mathrm{SE}=0.02, P<1 \times 10^{-16}\right)$ and $11.1-18.4 \%$ of the variance in region-wise

296 brain age gaps (all $P<2 \times 10^{-9}$ ).

Next, we assessed the overlap between the genetic underpinnings of brain age gap and common brain disorders. Focusing on those disorders that showed a significant brain age gap in the main analysis, we gathered genome-wide association analysis (GWAS) summary statistics for

300 SZ and BD from the Psychiatric Genomics Consortium ${ }^{30,31}$, MS from the International Multiple

301 Sclerosis Genetics Consortium ${ }^{32}$, and AD from the International Genomics of Alzheimer's

302 Project $^{33}$. In addition, we performed GWAS on the full brain and region-wise brain age gaps in

303 the above-described set of 16,269 healthy controls. We used conditional Q-Q plots ${ }^{34}$ to assess

304 polygenic overlap between two complex traits, conditioning GWAS summary statistics from each

305 of the brain age gaps on GWAS summary statistics from each of the disorders. Notably, our

306 results indicate genetic overlap between brain age gap and brain disorders. Figure 5b provides

307 exemplary illustrations of conditional Q-Q plots for the frontal brain age gap stratified by SZ, the

308 cingulate brain age gap stratified by $\mathrm{BD}$ and the full brain age gap stratified by MS. When

309 selecting subsets of SNPs based on their associations with the disorders, the nominal $-\log 10$

310 transformed P-values of the brain age gaps deviated from the trajectories expected under the

311 global null hypothesis, indicating that the brain age gaps are enriched for SNP associations with

312 the relevant disorder. SZ and MS also showed patterns of enrichment with subcortical brain age

313 gap and BD with frontal brain age gap, whereas no clear patterns were observed for AD (Suppl.

314 Fig. 6). 
Kaufmann et al., Genetics of brain age suggest an overlap with common brain disorders conjunctional FDR analyses ${ }^{35,36}$, to identify SNPs that are associated with both phenotypes. We

317 found 15 independent, significant loci showing pleiotropy between brain age gaps and SZ (2

318 occipital, 4 frontal, 3 temporal, 1 parietal, 2 cingulate, 1 insula, 2 cerebellar/subcortical; 116

319 SNPs in total), 6 loci for BD (3 frontal, 2 cingulate, 1 insula; 40 SNPs in total), 7 loci for MS (2

320 full brain, 2 frontal, 1 temporal, 2 subcortical; 7 SNPs in total) and 1 locus for AD (temporal, 1

321 SNP), respectively (Suppl. Table 3). An intronic variant (rs940904) in protein coding gene

322 PITPNM2 at chromosome 12q24.31 underlying the frontal brain age gap significantly overlapped

323 both with SZ and MS.

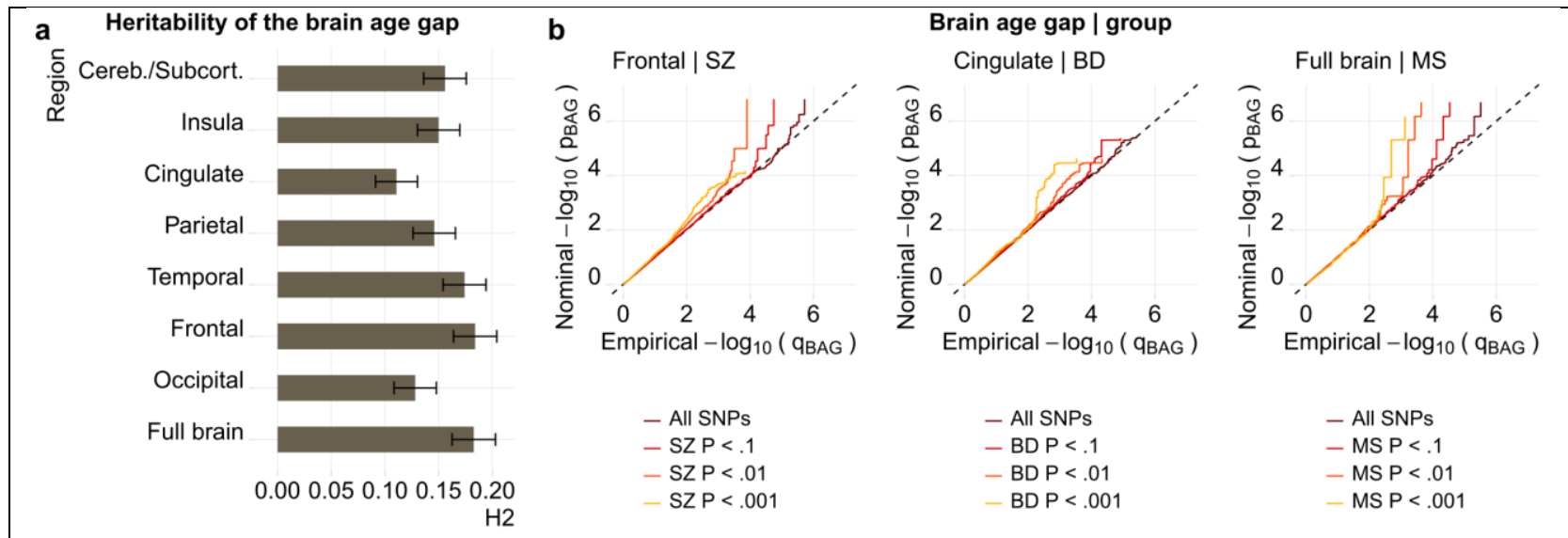

Fig. 5: The brain age gaps are heritable and the genetic underpinnings overlap with those observed for several disorders. a, Heritability (H2) estimated using GCTA (all $P<2 \times 10^{-9}$ ). b, Exemplary illustration of genetic enrichment between brain age gaps and SZ, BD and MS, assessed using conditional Q-Q plots. The dashed line is the expected trajectory under the global null hypothesis, whereas the coloured lines are the trajectories observed in the complete set, and in subsets of SNPs identified by their association with the disorder. Abbreviations: BAG, brain age gap. SNP, single nucleotide polymorphism.

\section{Discussion}

325 Taken together, our results provide strong evidence that several common brain disorders are 326 associated with accentuated aging of the brain compared to chronological age, with effects

327 observed in SZ, PSYMIX, BD, MS, MDD, MCI and DEM; but not in ASD, ADHD or SZRISK. 
Kaufmann et al., Genetics of brain age suggest an overlap with common brain disorders

328 Importantly, we revealed a distinct neuroanatomical distribution of brain age gaps in several

329 disorders. Associations with clinical and cognitive data underlined the functional relevance of the

330 brain age gaps and genetic analyses in healthy controls provided evidence that the brain age gaps

331 are heritable, with overlapping genes implicated in the genetic underpinnings of brain age gaps

332 and common brain disorders.

Our approach of estimating brain age at the level of brain regions was useful to reveal

334 differential spatial patterns between disorders. Whereas the implicated regions in the spatial brain

335 age profiles of the disorders matched previously reported structural and functional abnormalities

336 (e.g. frontal in $\mathrm{SZ}^{37-39}$, or the widespread volume loss in $\mathrm{AD}$ with large effects in subcortical

337 structures $^{40}$ ), our region-wise brain age approach preserved the well-established benefit of down-

338 sampling a large number of brain imaging features into a highly condensed and interpretable

339 score without a total loss of spatial sensitivity. As such, the analysis revealed substantial

340 differences in spatial aging profiles between disorders typically regarded neurodegenerative

341 disorders (MS, MCI, DEM) and disorders with established neurodevelopmental sources,

342 especially SZ and PSYMIX. Whereas these disorders were all associated with an increased brain

343 age gap on the full brain level, the region-wise analysis uncovered an interaction between the

344 frontal brain age patterns observed in SZ and PSYMIX and the cerebellar/subcortical patterns

345 observed in MS and DEM. Moreover, brain age gaps covered functional relevance beyond the

346 group differences. We identified significant associations with clinical and cognitive data, in

347 particular with scores of the Global Assessment of Functioning scale ${ }^{25}$ in SZ, with the Expanded

348 Disability Status Scale ${ }^{27}$ in MS and with Mini Mental State Examination scores ${ }^{28}$ in the dementia

349 spectrum. These results may warrant further research in which the link between the rate of

350 changes in brain age gaps and the clinical and cognitive outcome can be studied in a longitudinal

351 setting. Depending on the sensitivity of such associations to dynamic changes in clinical and 
Kaufmann et al., Genetics of brain age suggest an overlap with common brain disorders

352 cognitive function, such studies may also explore the biological mechanisms underlying these

353 associations as a potential anchor point for treatment options.

354 Genetic analysis offers one way of exploring the constraining factors underlying

355 phenotypic variation. Here, we provided evidence that full and region-wise brain age gaps

356 represent genetically influenced traits, and illustrated that the genetic variants associated with

357 brain age gaps are also associated with SZ, BD, MS and AD. In line with the accumulating

358 evidence that common disorders of the brain are highly polygenic and partly overlapping ${ }^{30-34,36,41}$,

359 these results suggest shared molecular genetic mechanisms between brain age gaps and brain

360 disorders. Statistical associations do not necessarily signify causation, and functional

361 interpretations of the identified genes should be made with caution. Larger imaging genetics

362 samples, in particular those including individuals with common brain disorders, may in the future

363 allow the investigation of specificity of the implicated genes. Considering the observed

364 interaction effects in spatial brain age profiles between some disorders, we speculate that such

365 analyses may offer novel insight into specific molecular mechanisms and will allow us to

366 delineate the processes that affect the pace and profile of global and regional brain aging for each

367 of these disorders.

368 In conclusion, in this largest brain age study to date, we established that the brain age gap

369 is genetically constrained, increased in several common brain disorders, and linked to clinical and

370 cognitive phenotypes. Our results establish the potential of advanced lifespan modelling in the

371 clinical neurosciences, highlighting the benefit of big data resources that cover a wide span of

372 ages and disease conditions. Delineating dynamic lifespan trajectories within and across

373 individuals will be essential to disentangle the pathophysiological complexity of brain disorders. 
Kaufmann et al., Genetics of brain age suggest an overlap with common brain disorders

\section{Acknowledgements}

The authors were funded by the Research Council of Norway (213837, 223273, 204966/F20, 229129, 249795/F20, 276082), the South-Eastern Norway Regional Health Authority (2013-123, 2014-097, 2015-073), Stiftelsen Kristian Gerhard Jebsen, and the European Commission 7th Framework Programme (602450, IMAGEMEND). The data used in this study were gathered from various sources. A detailed overview of the included cohorts and acknowledgement of their respective funding sources and cohort-specific details is provided in Supplementary Table 1.

\section{Author contributions}

T.K. and L.T.W. conceived the study; T.K., N.T.D. and L.T.W. pre-processed all data in Freesurfer; N.T.D., M.J.L., C.L.B, L.B.N., L.T.W. and T.K. performed quality control of the data; T.K. performed the analysis with contributions from L.T.W. and D.v.d.M.; T.K., L.T.W., N.T.D., D.v.d.M. and O.A.A. contributed to interpretation of the results. All remaining authors were involved in data collection at various sites as well as cohort-specific tasks. T.K. and L.T.W. wrote the first draft of the paper and all authors contributed to and approved the final manuscript.

\section{Competing financial interests}

Some authors received educational speaker's honorarium from Lundbeck (O.A. Andreassen, A. Bertolino, T. Elvsåshagen, M. Zink, N. I. Landrø), Sunovion (O.A. Andreassen), Shire (B. Franke), Medice (B. Franke), Otsuka (A. Bertolino, M. Zink) and Jannsen (A. Bertolino), Roche (M. Zink), Ferrer (M. Zink), Trommsdorff (M. Zink), Servier (M. Zink), all of these unrelated to this work. A. Bertolino is a stockholder of Hoffmann-La Roche Ltd and has received consultant fees from Biogen Idec. E. G. Celius and H. F. Harbo have received travel support, honoraria for advice and lecturing from Almirall (Celius), Biogen Idec (both), Genzyme (both), Merck (both), Novartis(both), Roche (both), Sanofi-Aventis (both) and Teva (both). They have received 
Kaufmann et al., Genetics of brain age suggest an overlap with common brain disorders

unrestricted research grants from Novartis (Celius, Harbo), Biogen Idec (Celius) and Genzyme

399 (Celius). G. Pergola has been the academic supervisor of a Roche collaboration grant (years

400 2015-16) that funds his salary. None of the mentioned external parties had any role in the

401 analysis, writing or decision to publish this work. Other authors declare no competing financial 402 interests.

\section{Materials \& Correspondence}

404 The data incorporated in this work were gathered from various resources (see 405 acknowledgements). Material requests will need to be placed with individual PIs. Corresponding 406 authors Tobias Kaufmann (tobias.kaufmann@medisin.uio.no) and Lars T. Westlye 407 (l.t.westlye@ psykologi.uio.no)will provide additional detail upon correspondence.

\section{Collaborators}

409 Members of the Karolinska Schizophrenia Project (KaSP): Farde $\mathrm{L}^{1}$, Flyckt $\mathrm{L}^{1}$, Engberg $\mathrm{G}^{2}$, 410 Erhardt $\mathrm{S}^{2}$, Fatouros-Bergman $\mathrm{H}^{1}$, Cervenka $\mathrm{S}^{1}$, Schwieler L $\mathrm{L}^{2}$, Piehl $\mathrm{F}^{3}$, Agartz $\mathrm{I}^{1,4,5}$, Collste $\mathrm{K}^{1}$,

411 Victorsson $\mathrm{P}^{1}$, Malmqvist $\mathrm{A}^{2}$, Hedberg $\mathrm{M}^{2}$, Orhan $\mathrm{F}^{2}$

$412{ }^{1}$ Centre for Psychiatry Research, Department of Clinical Neuroscience, Karolinska Institutet, \&

413 Stockholm County Council, Stockholm, Sweden; ${ }^{2}$ Department of Physiology and Pharmacology,

414 Karolinska Institutet, Stockholm, Sweden; ${ }^{3}$ Neuroimmunology Unit, Department of Clinical 415 Neuroscience, Karolinska Institutet, Stockholm, Sweden; ${ }^{4}$ NORMENT, KG Jebsen Centre for Psychosis 416 Research, Division of Mental Health and Addiction, University of Oslo, Oslo, Norway; ${ }^{5}$ Department of 417 Psychiatry Research, Diakonhjemmet Hospital, Oslo, Norway. 
Kaufmann et al., Genetics of brain age suggest an overlap with common brain disorders

\section{References}

421 1. WHO. World Health Statistics 2016 (2016).

422 2. Ormel, J., et al. JAMA 272, 1741-1748 (1994).

$423 \quad 3 . \quad$ Insel, T.R. \& Cuthbert, B.N. Science 348, 499-500 (2015).

$424 \quad 4 . \quad$ Prince, M., et al. Lancet 370, 859-877 (2007).

425 5. Parikshak, N.N., Gandal, M.J. \& Geschwind, D.H. Nat Rev Genet 16, 441-458 (2015).

426 6. Marin, O. Nat Med 22, 1229-1238 (2016).

$427 \quad 7 . \quad$ Insel, T.R. Nature 468, 187-193 (2010).

$428 \quad 8 . \quad$ Liguori, M., et al. Neurol Sci 21, S825-829 (2000).

429 9. Aubert-Broche, B., et al. Neurology 83, 2140-2146 (2014).

$430 \quad$ 10. Hasin, D.S., et al. Jama Psychiat (2018).

431 11. Masters, C.L., et al. Nat Rev Dis Primers 1, 15056 (2015).

432 12. Kaufmann, T., et al. Nature neuroscience 20, 513-515 (2017).

433 13. Giedd, J.N. \& Rapoport, J.L. Neuron 67, 728-734 (2010).

434 14. Schmitz, T.W., Nathan Spreng, R. \& Alzheimer's Disease Neuroimaging, I. Nat Commun 7,

435

436

437

438

439

440

441

442

443

444

445

446

447

448

449

450

451

452

453

454

455

456

457

458

459 13249 (2016).

15. Frisoni, G.B., Fox, N.C., Jack, C.R., Jr., Scheltens, P. \& Thompson, P.M. Nat Rev Neurol 6, 67-77 (2010).

16. Dosenbach, N.U., et al. Science 329, 1358-1361 (2010).

17. Cole, J.H. \& Franke, K. Trends Neurosci 40, 681-690 (2017).

18. Editorial. Nat Med 24, 1 (2018).

19. Fischl, B., et al. Neuron 33, 341-355 (2002).

20. Ho, D., Imai, K., King, G. \& Stuart, E.A. Journal of Statistical Software 42, 1-28 (2011).

21. Glasser, M.F., et al. Nature 536, 171-178 (2016).

22. Chen, T. \& Guestrin, C. in Proceedings of the 22nd ACM SIGKDD International Conference on Knowledge Discovery and Data Mining 785-794 (ACM, San Francisco, California, USA, 2016).

23. Smith, S.M. \& Nichols, T.E. Neuron 97, 263-268 (2018).

24. Filzmoser, P. \& Gschwandtner, M. in Multivariate Outlier Detection Based on Robust Methods. $R$ package v 2.0.9 (2018).

25. Pedersen, G. \& Karterud, S. Comprehensive Psychiatry 53, 292-298 (2012).

26. Kay, S.R., Fiszbein, A. \& Opfer, L.A. Schizophrenia Bull 13, 261 (1987).

27. Kurtzke, J.F. Neurology 33, 1444-1444 (1983).

28. Folstein, M.F., Folstein, S.E. \& McHugh, P.R. Journal of psychiatric research 12, 189-198 (1975).

29. Yang, J., Lee, S.H., Goddard, M.E. \& Visscher, P.M. Am J Hum Genet 88, 76-82 (2011).

30. Ripke, S., et al. Nature 511, 421 (2014).

31. Stahl, E., et al. bioRxiv (2018).

32. Sawcer, S., et al. Nature 476, 214 (2011).

33. Lambert, J.-C., et al. Nature genetics 45, 1452 (2013).

34. Andreassen, O.A., et al. PLoS genetics 9, e1003455 (2013).

$460 \quad 35 . \quad$ Nichols, T., Brett, M., Andersson, J., Wager, T. \& Poline, J.-B. Neuroimage 25, 653-660 (2005).

461 36. $\quad$ Andreassen, O.A., et al. The American Journal of Human Genetics 92, 197-209 (2013).

462 37. Ellison-Wright, I. \& Bullmore, E. Schizophrenia research 117, 1-12 (2010).

463 38. Moberget, T., et al. Molecular psychiatry (2017).

464 39. Pettersson-Yeo, W., Allen, P., Benetti, S., McGuire, P. \& Mechelli, A. Neurosci Biobehav R 35,

465 1110-1124 (2011).

466 40. Jernigan, T.L., Salmon, D.P., Butters, N. \& Hesselink, J.R. Biological psychiatry 29, 68-81

$467 \quad$ (1991).

468 41. Andreassen, O.A., et al. Molecular psychiatry 20, 207 (2015). 
Kaufmann et al., Genetics of brain age suggest an overlap with common brain disorders

\section{Online methods}

$471 \quad$ Samples

472 We have included data collected through collaborations, data sharing platforms, consortia as well

473 as available in-house cohorts. Supplementary Table 1 and $\mathbf{2}$ provide detailed information on the

474 individual cohorts. All included cohorts have been published on, and we refer to a list of

475 publications that can be consulted for a more detailed overview of cohort characteristics. Data

476 collection in each cohort was performed with participants' written informed consent and with

477 approval by the respective local Institutional Review Boards.

\section{$478 \quad$ Image pre-processing and quality control}

479 Raw T1 data for all study participants were stored and analysed locally at University of Oslo,

480 following a harmonized analysis protocol applied to each individual subject data (Suppl. Fig. 1).

481 We performed automated surface-based morphometry and subcortical segmentation using

482 Freesurfer $5.3^{19}$. We deployed an automated quality control protocol executed within each of the

483 contributing cohorts (Suppl. Tables 1-2), that excluded potential outliers based on global data

484 quality measures. In brief, we regressed age, age ${ }^{2}$, sex and (in case of multiple scanners) scanning

485 site from mean cortical thickness, cortex volume, subcortical grey matter volume and from

486 estimated total intracranial volume. Next, we z-standardized the resulting absolute of the

487 residuals and excluded those subjects that exceeded a pre-defined standard deviation (SD)

488 threshold of 4 SD. On top of this, a random set of data was carefully screened by trained research

489 personnel to identify segmentation errors, assess the quality of each subject's brain images

490 manually, edit segmentation where possible and to exclude data of insufficient quality $(n=3957$

491 manually controlled, $n=166$ excluded, $n=219$ edited). Taken together, the main analysis

492 excluded cases identified by manual QC as well as cases exceeding a threshold of 4 SD in the 
Kaufmann et al., Genetics of brain age suggest an overlap with common brain disorders

493 automated quality control on either of the four brain imaging measures, yielding 36,891 subjects.

494 In addition, we performed supplementary analyses using a subset of data, where a more stringent

495 quality control and exclusion procedure was applied. Using multivariate outlier detection based

496 on robust methods as implemented in the $\mathrm{R}$ package mvoutlier ${ }^{24}$, we identified an additional 5513

497 data sets with potentially less sufficient data quality. Thus, supplemental analysis provides a

498 sanity check with those subjects excluded (sample size: $n=31,378$ ).

\section{Brain age prediction}

500 We utilized the most recent cortical parcellation scheme ${ }^{21}$ to extract cortical thickness, area and

501 volume for 180 regions of interest (ROI) per hemisphere. In addition, we extracted the classic set

502 of cerebellar/subcortical and cortical summary statistics ${ }^{19}$. This yielded a total set of 1118

503 structural brain imaging features (360/360/360/38 for cortical thickness/area/volume as well as

504 cerebellar/subcortical and cortical summary statistics, respectively).

We used machine learning on this feature set to predict the age of each individual's brain.

506 First, we split the available data into a training sample and ten independent test samples, as

507 described in the main text (Fig. 1a). Next, for each sex, we trained machine learning models

508 utilizing the xgboost package in $\mathrm{R}^{42}$, chosen due to its resource efficiency and demonstrated

509 superior performance in previous machine learning competitions, to predict the age of the brain

510 using data available in the training set. First, model parameters were tuned using a 5-fold cross-

511 validation of the training data. This step identified the optimal number of model training

512 iterations by assessing the prediction error for 1500 rounds and implementing an early stopping if

513 the performance did not improve for 20 rounds. Based on previous experience, the learning rate

514 was pre-set to eta=0.01 and all other parameters were set to default ${ }^{42}$ for linear $x g b o o s t$ tree

515 models. After determining the optimal number of training iterations, the full set of training data 
Kaufmann et al., Genetics of brain age suggest an overlap with common brain disorders

516 was used to train the final models with the adjusted nrounds parameter. In addition, to assess

517 overall model performance, prediction models were cross-validated within the training set using a

518 5-fold cross validation, each fold implementing the above described training procedure and

519 testing on the hold-out part of the training set. Brain age predictions on the level of individual

520 brain regions followed the same procedures as those described for the full brain level, except that

521 the feature set was reduced to cover only those features that overlapped more than $50 \%$ with a

522 given lobe. Regions were defined following the Freesurfer lobesStrict segmentation as occipital,

523 frontal, temporal, parietal, cingulate and insula. In addition, given the limited number of

524 cerebellar features available in the Freesurfer summary statistics, cerebellar and subcortical

525 features were grouped into a cerebellar/subcortical region (Fig. 1b).

526 For the genetic analyses, a different approach had to be taken to ensure maximum

527 exploitation of the limited availability of genetic data. Rather than splitting into training and test

528 sets, we selected all healthy subjects and estimated their brain age using a 5-fold cross-validation

529 approach like the one performed when validating performance of the training set. The resulting

530 unbiased estimates of brain age gaps for all individuals with genetic data available went into the

531 genome-wide complex trait analysis and conjunctional FDR.

\section{Main statistical analysis framework}

533 We performed both mega- (across cohorts) and meta- (within cohort) analyses. To estimate group

534 effects on a given measure in a mega-analysis framework, we computed the effect of diagnosis in

535 relation to the healthy controls for each of the ten test samples in a linear model accounting for

536 age, age ${ }^{2}$, sex and scanning site. Cohen's d effect sizes were estimated based on contrast t-

537 statistics $^{43}$ following Formula 1:

$$
d=\frac{t\left(n_{1}+n_{2}\right)}{\sqrt{n_{1} n_{2}} \sqrt{d f}}
$$


Kaufmann et al., Genetics of brain age suggest an overlap with common brain disorders

538 For the meta-analysis, similar models were computed within cohorts. In addition to estimating

539 Cohen's d (Formula 1), we estimated the variance of d following Formula 2.

$$
v=\left(\frac{n_{1}+n_{2}}{n_{1} n_{2}}+\frac{d^{2}}{2\left(n_{1}+n_{2}-2\right)}\right)\left(\frac{n_{1}+n_{2}}{n_{1}+n_{2}-2}\right)
$$

540 Cumulative effects across cohorts were then estimated using a variance-weighted random-effects

541 model as implemented in the metafor package in $\mathrm{R}^{44}$.

Assessment of regional specificity

543 The clustering in Figure 3b was performed using heatmap. 2 from the gplots package ${ }^{45}$ in R. A

544 correlation matrix was computed based on the case-control effect sizes obtained from each test

545 sample and region and hierarchical clustering was performed using the default settings. To

546 further explore regional specificity, we performed another analysis that involved only the clinical

547 groups. We regressed age, age ${ }^{2}$, sex and scanning site from the brain age gaps in each test

548 sample. Next, we joined data from each pair of clinical groups and each pair of regions for

549 repeated measures analysis of variance and estimated the effect sizes of region $\mathrm{x}$ group

550 interactions (1260 ANOVAs in total). The interaction effects were visualized in Figure 3c using

551 the circlize package ${ }^{46}$ in $\mathrm{R}$.

\section{Genetic analyses}

553 We restricted all genetic analyses to individuals with European ancestry, as determined through

554 multidimensional scaling (MDS), and included the first four population components as covariates

555 to further control for population stratification. Single Nucleotide Polymorphism (SNP) data were

556 available for 16,269 adult healthy individuals with European ancestry. We used genome-wide

557 complex trait analysis ${ }^{29}$ (GCTA) to estimate the proportion of variance in brain age explained by

558 SNPs. Before the analysis, we removed high LD regions from the genetic data and pruned it,

559 using a sliding window approach with a window size of 50 base pair (bp), a step size of 5 bp and 
Kaufmann et al., Genetics of brain age suggest an overlap with common brain disorders

560 an $\mathrm{r}^{2}$ of 0.2 , leaving 133,147 SNPs. All GCTA analyses accounted for age, age ${ }^{2}$, sex, scanning

561 site and genetic batch.

562

Furthermore, we used conditional Q-Q plots $^{34}$ and conjunctional FDR analyses ${ }^{35,36}$ to

563 assess polygenic overlap between two complex traits. We gathered genome-wide association

564 analysis (GWAS) summary statistics for SZ and BD from the Psychiatric Genomics

565 Consortium ${ }^{30,31}$, MS from the International Multiple Sclerosis Genetics Consortium ${ }^{32}$, and AD

566 from the International Genomics of Alzheimer's Project ${ }^{33}$; and performed GWAS on the full

567 brain and region-wise brain age gaps in the above-described sample of 16,269 healthy adults. The

568 MHC region was excluded from the analysis. The SNPs were pruned using a pairwise correlation

569 coefficient approximation to LD $\left(\mathrm{r}^{2}\right)$, where SNPs were disregarded at $\mathrm{r}^{2}<0.2$ and pruning

570 performed with 20 iterations, as described elsewhere ${ }^{34}$. Conjunctional FDR was run for each pair

571 of full brain / region-wise brain age gap and group, using conjunctional FDR threshold of 0.05.

572 SNPs were annotated using the Ensembl Variant Effect Predictor ${ }^{47}$.

573 Cognitive and clinical associations

574 Cognitive and clinical associations were tested in subsets based on data availability as described

575 in the main text. First, we regressed age, age ${ }^{2}$, sex and scanning site from the brain age gaps.

576 Next, we correlated the resulting residuals with scores of the Global Assessment of Functioning

577 scale $^{25}$ (GAF), the Positive and Negative Syndrome Scale ${ }^{26}$ (PANSS), the Expanded Disability

578 Status Scale ${ }^{27}$ (EDSS) and Mini Mental State Examination scores ${ }^{28}$ (MMSE). We transformed

579 the resulting correlations using Fisher's $\mathrm{z}$ transform. Therefore, the reported associations

580 essentially reflect a partial correlation between full brain / region-wise brain age gaps and

581 clinical/cognitive scores, controlling for confounding effects of age, sex and site.

\section{Code availability.}


Kaufmann et al., Genetics of brain age suggest an overlap with common brain disorders

The main analysis was performed using $\mathrm{R}$ statistics ${ }^{48}$. The code needed to reproduce the results is

584 available from the authors upon request.

\section{References of the Supplement}

19. Fischl, B., et al. Neuron 33, 341-355 (2002).

21. Glasser, M.F., et al. Nature 536, 171-178 (2016).

24. Filzmoser, P. \& Gschwandtner, M. in Multivariate Outlier Detection Based on Robust Methods. $R$ package v 2.0.9 (2018).

25. Pedersen, G. \& Karterud, S. Comprehensive Psychiatry 53, 292-298 (2012).

592

26. Kay, S.R., Fiszbein, A. \& Opfer, L.A. Schizophrenia Bull 13, 261 (1987).

27. Kurtzke, J.F. Neurology 33, 1444-1444 (1983).

28. Folstein, M.F., Folstein, S.E. \& McHugh, P.R. Journal of psychiatric research 12, 189-198 (1975).

29. Yang, J., Lee, S.H., Goddard, M.E. \& Visscher, P.M. Am J Hum Genet 88, 76-82 (2011).

30. Ripke, S., et al. Nature 511, 421 (2014).

31. Stahl, E., et al. bioRxiv (2018).

32. Sawcer, S., et al. Nature 476, 214 (2011).

598 33. Lambert, J.-C., et al. Nature genetics 45, 1452 (2013).

599 34. Andreassen, O.A., et al. PLoS genetics 9, e1003455 (2013).

$60035 . \quad$ Nichols, T., Brett, M., Andersson, J., Wager, T. \& Poline, J.-B. Neuroimage 25, 653-660 (2005).

601 36. Andreassen, O.A., et al. The American Journal of Human Genetics 92, 197-209 (2013).

602 42. Tianqi, C., Tong, H., Benesty, M., Khotilovich, V. \& Tang, Y. in Xgboost: extreme gradient

$603 \quad$ boosting. R package v0.4-2 (2015).

604 43. Nakagawa, S. \& Cuthill, I.C. Biol Rev Camb Philos Soc 82, 591-605 (2007).

605 44. Viechtbauer, W. Journal of Statistical Software 36, 1-48 (2010).

606

46. Gu, Z. in $R$ Package circlize: Circular Visualization (2017).

47. McLaren, W., et al. Genome biology 17, 122 (2016).

48. R Core Team. R Foundation for Statistical Computing, Vienna, Austria. (2013).

49. Di Martino, A., et al. Molecular psychiatry 19, 659-667 (2014).

50. Di Martino, A., et al. Sci Data 4, 170010 (2017).

51. Liu, Y., et al. Neurobiol Aging 32, 1198-1206 (2011).

52. Lovestone, S., Francis, P. \& Strandgaard, K. J Nutr Health Aging 11, 359-361 (2007).

53. Brown, M.R., et al. Front Syst Neurosci 6, 69 (2012).

54. Consortium, H.D. Front Syst Neurosci 6, 62 (2012).

55. Guadalupe, T., et al. Brain Imaging Behav 11, 1497-1514 (2017).

56. Conzelmann, A., et al. Biological psychiatry 65, 578-585 (2009).

57. Weiner, M.W., et al. Alzheimers Dement 6, 202-211 e207 (2010).

58. Wyman, B.T., et al. Alzheimers Dement 9, 332-337 (2013).

59. Nilsson, L.-G., et al. Aging, Neuropsychology, and Cognition 11, 134-148 (2004).

60. Taylor, J.R., et al. Neuroimage 144, 262-269 (2017).

61. Shafto, M.A., et al. BMC Neurol 14, 204 (2014).

62. Eisenacher, S., et al. Psychol Med 45, 3329-3340 (2015).

63. Rausch, F., et al. Schizophrenia research 156, 143-149 (2014).

64. Zuo, X.N., et al. Sci Data 1, 140049 (2014).

65. Lu, H., et al. Cereb Cortex 21, 1426-1434 (2011).

66. Gorgolewski, K.J., Durnez, J. \& Poldrack, R.A. F1000Res 6, 1262 (2017).

67. Poldrack, R.A., et al. Sci Data 3, 160110 (2016).

629 68. Repovs, G. \& Barch, D.M. Frontiers in human neuroscience 6, 137 (2012). 
Kaufmann et al., Genetics of brain age suggest an overlap with common brain disorders

630 69. Repovs, G., Csernansky, J.G. \& Barch, D.M. Biological psychiatry 69, 967-973 (2011).

631 70. Velanova, K., Wheeler, M.E. \& Luna, B. Cereb Cortex 18, 2505-2522 (2008).

632 71. Lepping, R.J., Ruth, A.A. \& Cary, R. Psychology of music 44 (2016).

633 72. Van Schuerbeek, P., Baeken, C. \& De Mey, J. PloS one 11, e0153865 (2016).

634 73. Van Schuerbeek, P., Baeken, C., De Raedt, R., De Mey, J. \& Luypaert, R. Brain Res 1371, $32-42$

635

636

637

638

639

640

641

642

643

644

645

646

647

648

649 (2011).

74. FitzGerald, T.H.B., Hammerer, D., Friston, K.J., Li, S.C. \& Dolan, R.J. PLoS Comput Biol 13, e1005418 (2017).

75. Van Essen, D.C., et al. Neuroimage 80, 62-79 (2013).

76. Haukvik, U.K., et al. Psychol Med 42, 1329-1337 (2012).

77. Haberg, A.K., et al. PloS one 11, e0151080 (2016).

78. Krokstad, S., et al. Int J Epidemiol 42, 968-977 (2013).

79. Liu, K., et al. Front Aging Neurosci 9, 275 (2017).

80. Collste, K., et al. Molecular psychiatry 22, 850-856 (2017).

81. Orhan, F., et al. Molecular psychiatry (2017).

82. Elvsashagen, T., et al. Bipolar disorders 15, 167-176 (2013).

83. Elvsashagen, T., et al. Bipolar disorders 15, 855-864 (2013).

84. Espeseth, T., et al. Twin Res Hum Genet 15, $442-452$ (2012).

85. von Rhein, D., et al. Eur Child Adolesc Psychiatry 24, 265-281 (2015).

Buckner, R.L., et al. Neuroimage 23, 724-738 (2004)

651 88. Fotenos, A.F., Snyder, A.Z., Girton, L.E., Morris, J.C. \& Buckner, R.L. Neurology 64, 1032-1039

652 (2005).

653 89. Jernigan, T.L., et al. Neuroimage 124, 1149-1154 (2016).

654 90. Satterthwaite, T.D., et al. Neuroimage 124, 1115-1119 (2016).

655 91. Satterthwaite, T.D., et al. Neuroimage 86, 544-553 (2014).

656 92. Sowa, P., et al. Mult Scler, 1352458518765671 (2018).

657 93. Wei, D., et al. bioRxiv (2018).

658 94. Bustillo, J.R., et al. Schizophr Bull 43, 611-619 (2017).

659 95. Cetin, M.S., et al. Neuroimage 97, 117-126 (2014).

660 96. Gollub, R.L., et al. Neuroinformatics 11, 367-388 (2013).

661 97. Kogan, A., Alpert, K., Ambite, J.L., Marcus, D.S. \& Wang, L. Neuroimage 124, 1196-1201 (2016).

662 98. Wang, L., et al. Neuroimage 124, 1155-1167 (2016).

663 99. Ambite, J.L., et al. Data Integr Life Sci 9162, 37-51 (2015).

664 100. Borgwardt, S., et al. Schizophr Bull 39, 1105-1114 (2013).

665 101. Dukart, J., et al. Journal of psychiatry \& neuroscience : JPN 42, 307-319 (2017).

666 102. Wang, Y., Wei, D., Li, W. \& Qiu, J. Neuroscience 272, 217-228 (2014).

667 103. Zhu, W., et al. Brain Cogn 103, 70-76 (2016).

668 104. Dorum, E.S., et al. Brain Behav 6, e00533 (2016).

669 105. Kaufmann, T., et al. Neuroimage 147, 243-252 (2016).

670 106. Kaufmann, T., et al. Schizophr Bull (2015).

671

672

673

674

675

107. Skåtun, K.C., et al. Journal of psychiatry \& neuroscience : JPN 41, 150159 (2016).

108. Brandt, C.L., et al. Schizophr Bull (2015).

109. Heck, A., et al. Neuron 81, 1203-1213 (2014).

110. Alfaro-Almagro, F., et al. Neuroimage 166, 400-424 (2018).

111. Pergola, G., et al. Schizophrenia research 180, 13-20 (2017).

676

677 
Kaufmann et al., Genetics of brain age suggest an overlap with common brain disorders

\section{Supplementary Figures}

1. Data sharing platforms $\rightarrow$ Structural magnetic

$$
\& \text { collaborations resonance images (raw data) }
$$

2. Unified pre-processing pipeline

(run at University of Oslo)

- Full brain reconstruction (Freesurfer)

- Extraction of cortical features from Human Connectome

Project atlas and cerebellar/subcortical features

- Quality control

3. Split data into a training and ten test sets

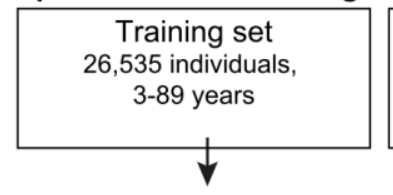

4. Train and cross validate age prediction models

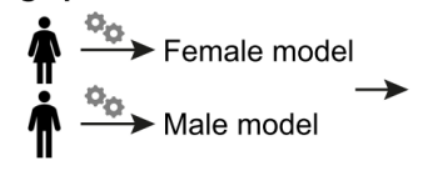

6. Statistical analysis, assessing group differences

in brain age gap

- Mega analysis (across-) and meta analysis (within-samples)

- Effect of diagnosis, accounting for age, $\mathrm{age}^{2}$, sex and scanning site

Suppl. Figure 1: Outline of the main analysis pipeline. 
Kaufmann et al., Genetics of brain age suggest an overlap with common brain disorders

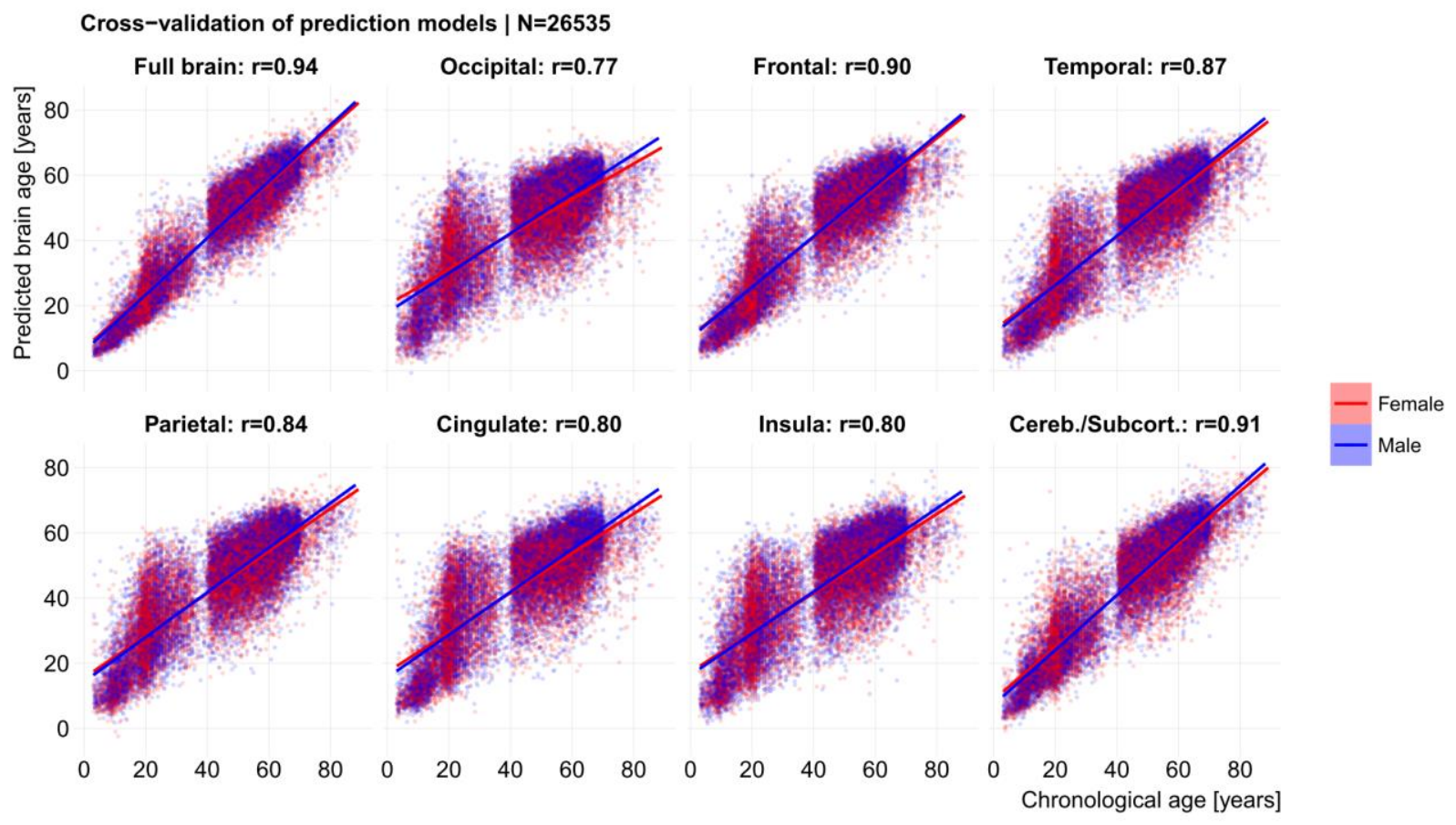

Suppl. Figure 2: Cross-validation of the prediction models confirmed validity of the models. The correlation between chronological age and predicted brain age estimated using 5-fold cross-validation within the training set is shown for each feature set. 
bioRxiv preprint doi: https://doi.org/10.1101/303164; this version posted April 17, 2018. The copyright holder for this preprint (which was not certified by peer review) is the author/funder. All rights reserved. No reuse allowed without permission.

Kaufmann et al., Genetics of brain age suggest an overlap with common brain disorders

683

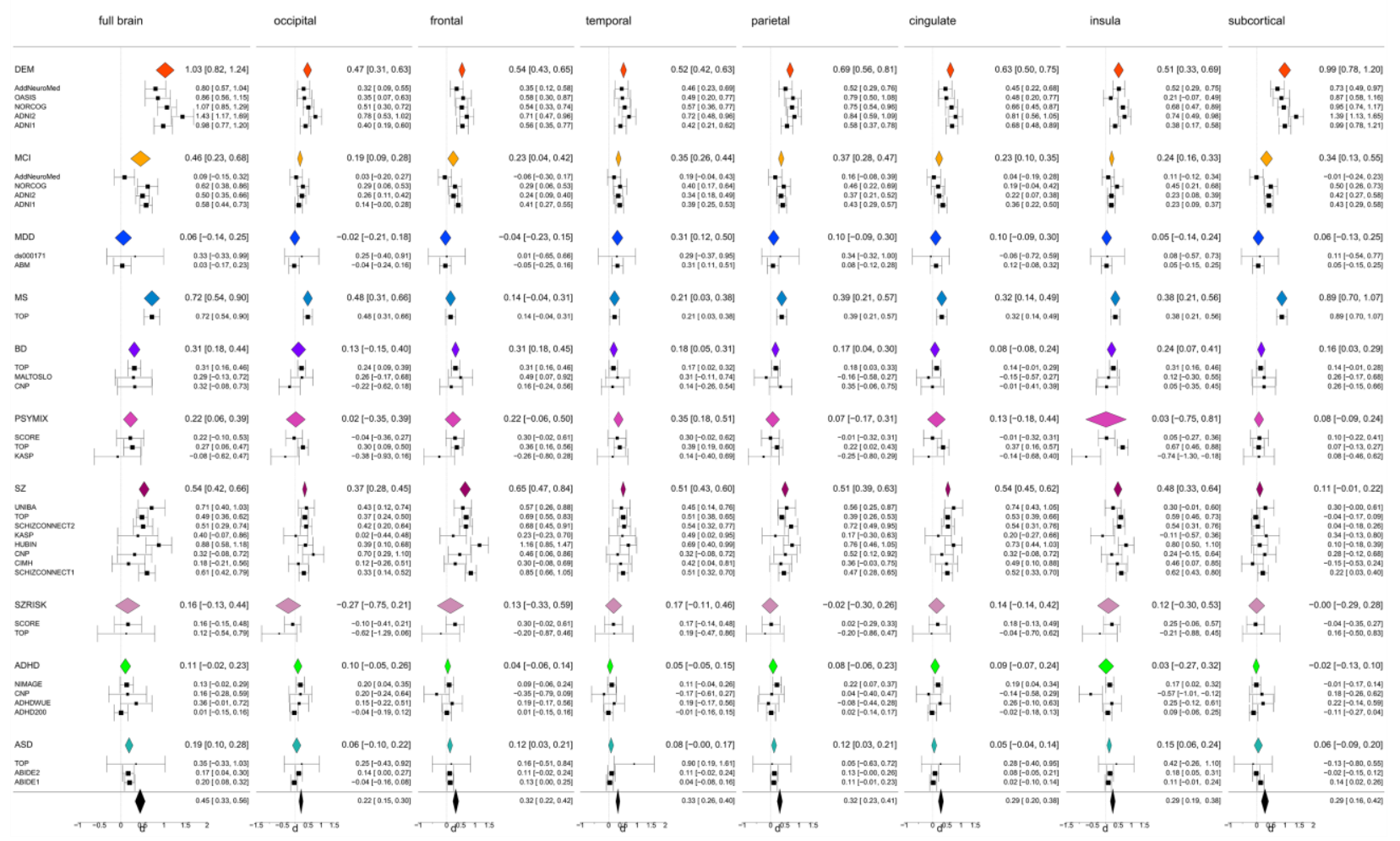

Suppl. Figure 3: Meta-analysis confirmed mega-analysis results. All Cohen's d effect sizes for the effect of group accounted for age, age ${ }^{2}$ and sex. Further, Cohen's $d$ for all cohorts that were collected at multiple sites also accounted for scanning site. 
bioRxiv preprint doi: https://doi.org/10.1101/303164; this version posted April 17, 2018. The copyright holder for this preprint (which was not certified by peer review) is the author/funder. All rights reserved. No reuse allowed without permission.

Kaufmann et al., Genetics of brain age suggest an overlap with common brain disorders
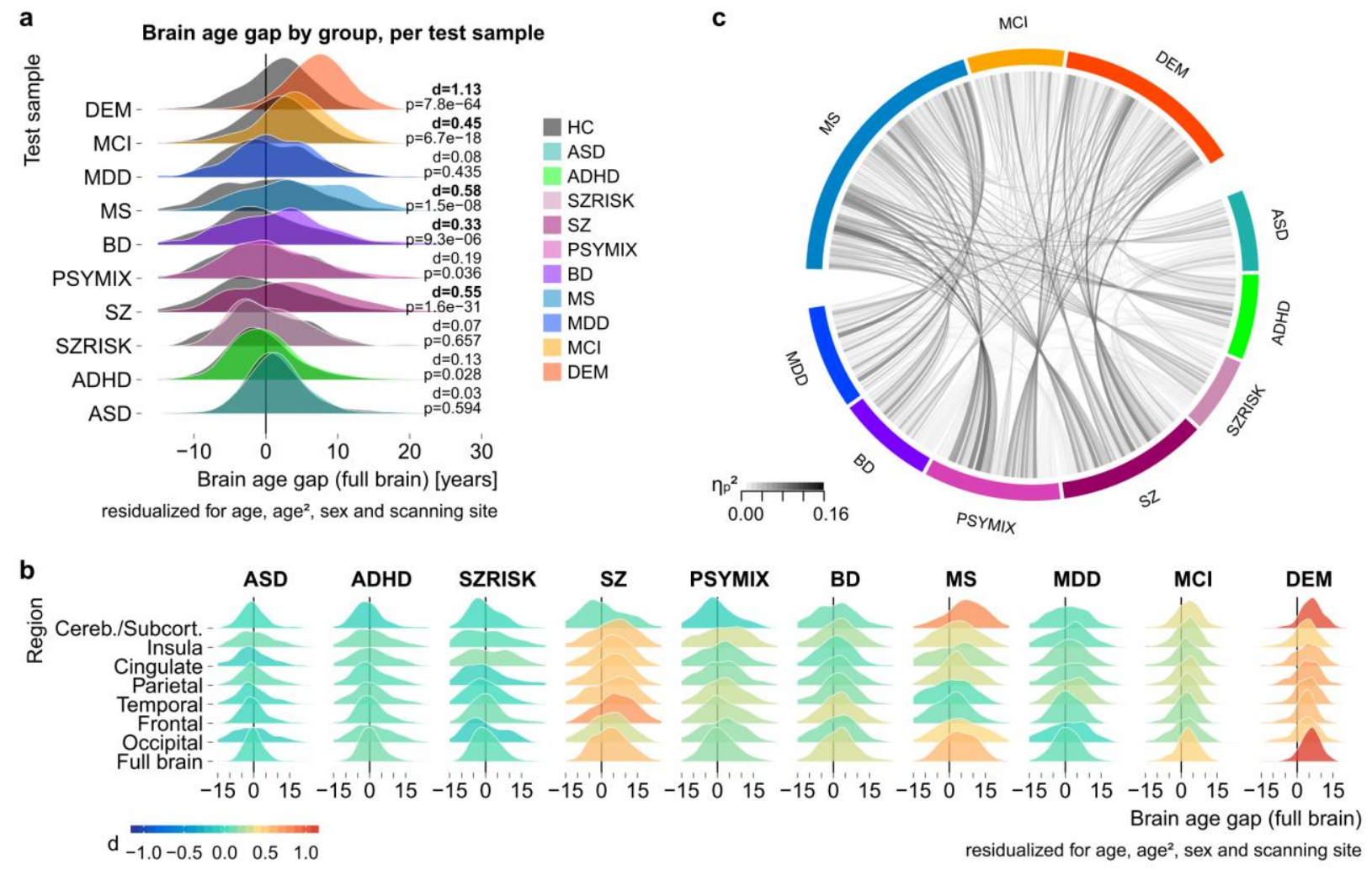

d
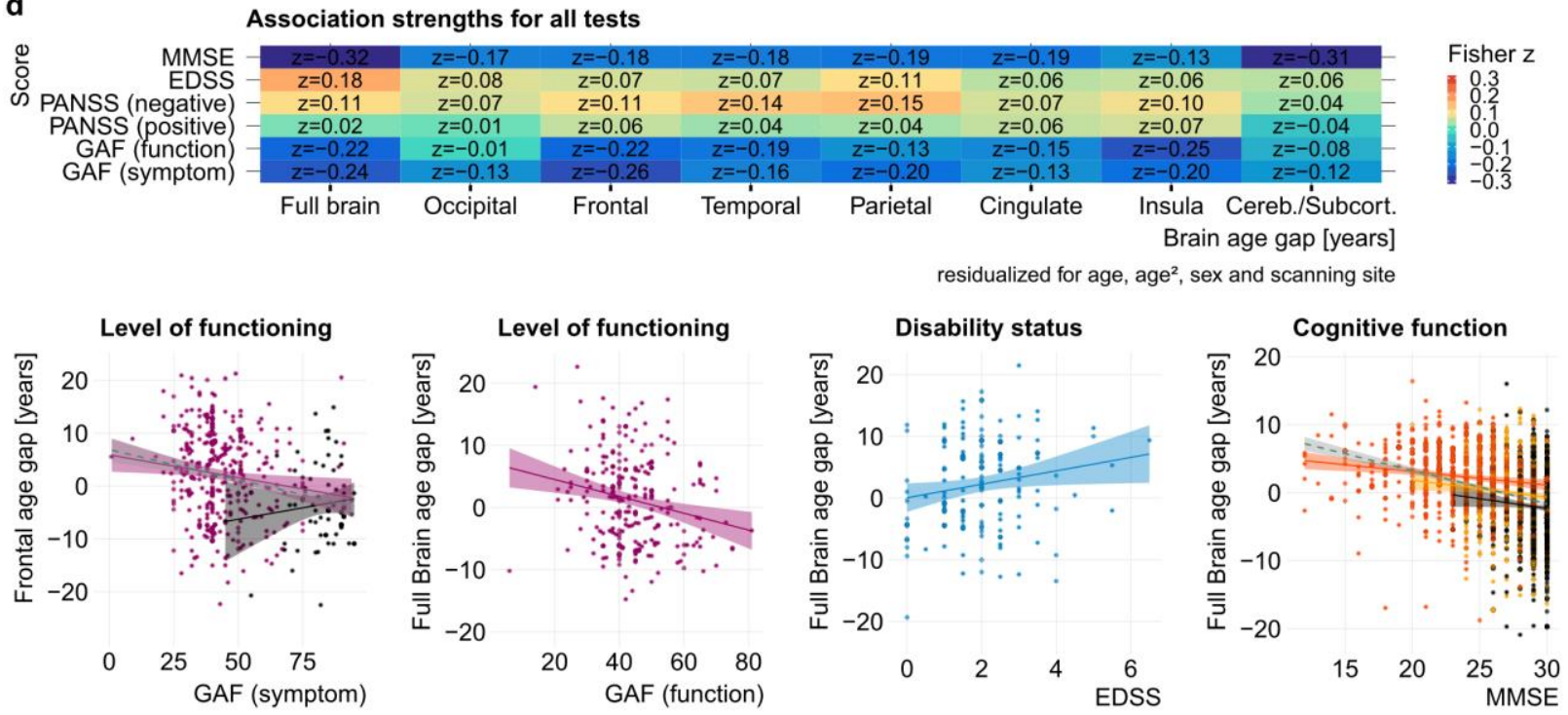

$-\mathrm{HC}-\mathrm{SZ}-\mathrm{MS}-\mathrm{MCl}-\mathrm{DEM}-$ Across groups

\section{Suppl. Figure 4: Replication of results in a subset of 31,378 individuals following more stringent,} multivariate exclusion criteria. a, Replication of group effects (for comparison, see Fig. 2) b, Replication of spatial brain age gap patterns (for comparison, see Fig. 3a) c, Replication of interaction effect pattern (for comparison, see Fig 3c). d, Replication of associations with clinical and cognitive scores (for comparison, see Fig. 4). 
bioRxiv preprint doi: https://doi.org/10.1101/303164; this version posted April 17, 2018. The copyright holder for this preprint (which was not certified by peer review) is the author/funder. All rights reserved. No reuse allowed without permission.

Kaufmann et al., Genetics of brain age suggest an overlap with common brain disorders

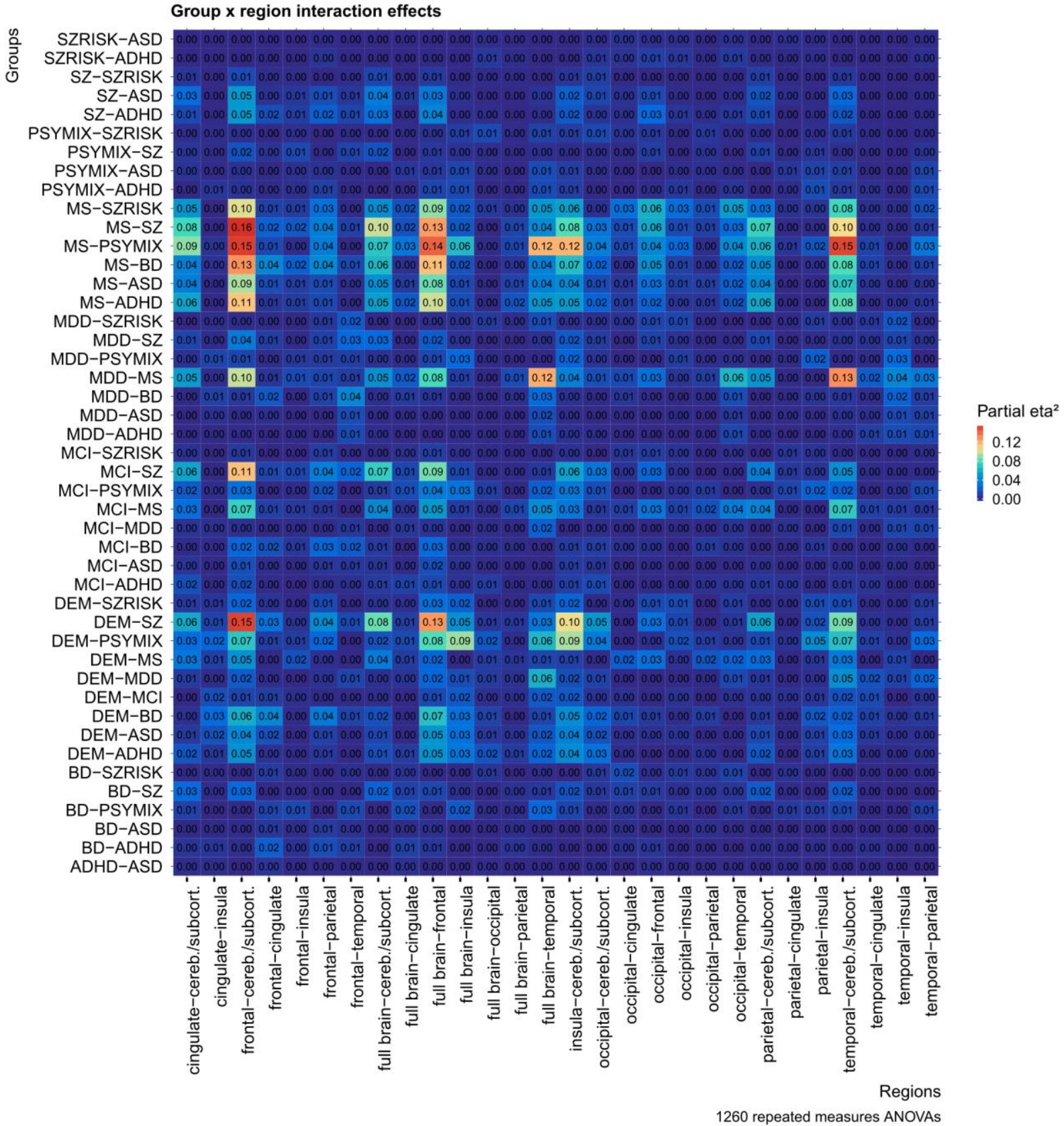

\section{Suppl. Figure 5: Results from 1260 repeated measures ANOVAs confirm group x region interaction}

effects in brain age patterns. Strongest effects were observed between MS and SZ, MS and PSYMIX as well as DEM and SZ, suggesting divergent aging patterns in these disorders. 
Kaufmann et al., Genetics of brain age suggest an overlap with common brain disorders
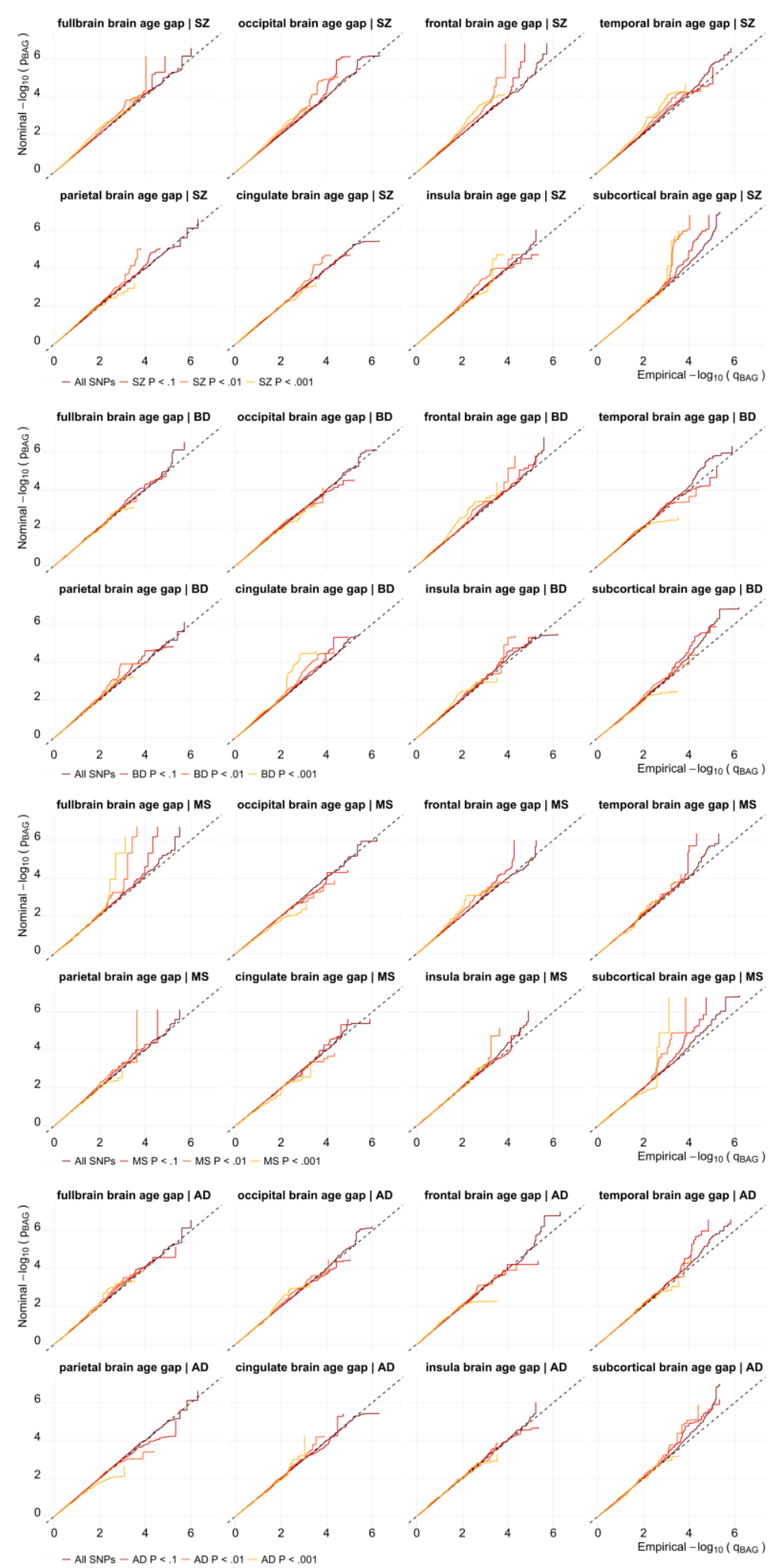
bioRxiv preprint doi: https://doi.org/10.1101/303164; this version posted April 17, 2018. The copyright holder for this preprint (which was not certified by peer review) is the author/funder. All rights reserved. No reuse allowed without permission.

\section{Kaufmann et al., Genetics of brain age suggest an overlap with common brain disorders}

\section{Supplementary Tables}

690 Suppl. Table 1: Summary of the included cohorts. Several of the cohorts are under ongoing data 691 collection, thus the subject numbers provided in the reference publications may not match those in this

692 article.

\begin{tabular}{ll}
\hline Cohort & Source \\
\hline ABIDE1 & \\
& http://fcon_1000.projects.nitrc.org/
\end{tabular}

ABIDE2 Commen

Reference

Primary support for the work by Adriana Di Martino was provided by the NIMH (K23MH087770) and the Leon Levy Foundation. Primary support for the work by Michael P. Milham and the INDI team was provided by gifts from Joseph P. Healy and the Stavros Niarchos Foundation to the Child Mind Institute, as well as by an NIMH award to MPM (R03MH096321).

Primary support for the work by Adriana Di Martino and her team was provided by the National Institute of Mental Health (NIMH 5R21MH107045). Primary support for the work by Michael P. http://fcon_1000.projects.nitrc.org/ Milham and his team provided by the National Institute of Mental Health (NIMH 5R21MH107045); Nathan S. Kline Institute of Psychiatric Research). Additional Support was provided by gifts from Joseph P. Healey, Phyllis Green and Randolph Cowen to the Child Mind Institute.

ABM Authors

ABM was supported by the Research Council of Norway (grant number 229135) and Health South East Research Funding Agency (grant number 2015052)

AddNeuroMed consortium was led by Simon Lovestone, Bruno Vellas, Patrizia Mecocci, Magda Tsolaki, Iwona Kłoszewska, Hilkka Soininen. Their work was supported by InnoMed (Innovative Medicines in Europe), an integrated project funded by the European Union of the Sixth Framework program priority (FP62004- LIFESCIHEALTH-5)

ADHD200

F. Xavier Castellanos, David Kennedy, Michael Milham, and Stewart Mostofsky are responsible for the initial conception of the ADHD-200 Consortium. Consortium steering committee includes Jan Buitelaar, F. Xavier Castellanos, Dan Dickstein, Damien Fair, David Kennedy, Beatriz Luna, Michael Milham (Project Coordinator), Stewart Mostofsky, and Julie Schweitzer. Data http://fcon_1000.projects.nitrc.org/ aggregation and organization was coordinated by the INDI team, which included Saroja Bangaru, David Gutman, Maarten Mennes, and Michael Milham. Web infrastructure and data storage were coordinated by Robert Buccigrossi, Albert Crowley, Christian Hasselgrove, David Kennedy, Kimberly Pohland, and Nina Preuss. The ADHD-200 Global Competition Coordinators were Damien Fair (Chair of Selection Committee, Editor in Chief for Global Competition Special issue) and Michael Milham

Primary support for the study was provided by the German Research Foundation, grant number DFG KFO $125 \quad 1 / 2$ and Pa566/7-3. KPL and his team are supported by the Deutsche Forschungsgemeinschaft (DFG: CRU 125, CRC TRR 58 A1/A5), European Community (EC: AGGRESSOTYPE FP7/No. 602805; Fritz Thyssen Foundation (No. 10.13.1185), ERA-Net NEURON/RESPOND, No. 01EW1602B, and 5-100 Russian Academic Excellence Project.

Data used in the preparation of this article were obtained from the Alzheimer's Disease Neuroimaging Initiative (ADNI) database (adni.loni.usc.edu). The ADNI was launched in 2003 as a publicprivate partnership, led by Principal Investigator Michael W. Weiner, MD. The primary goal of ADNI has been to test whether serial magnetic resonance imaging (MRI), positron emission tomography (PET), other biological markers, and clinical and neuropsychological assessment can be combined to measure the progression of mild cognitive impairment (MCI) and early Alzheimer's disease (AD). For up-to-date information, see www.adni-info.org.

Data collection and sharing for this project was funded by the Alzheimer's Disease Neuroimaging Initiative (ADNI) (National Institutes of Health Grant U01 AG024904) and DOD ADNI (Department of Defense award number W81XWH-12-2-0012). ADNI is funded by the National Institute on Aging, the National Institute of Biomedical Imaging and Bioengineering, and through generous contributions from the following: AbbVie, Alzheimer's Association; Alzheimer's Drug Discovery Foundation; Araclon Biotech; BioClinica, Inc.; Biogen; Bristol-Myers Squibb Company; CereSpir, Inc.; Cogstate; Eisai Inc.; Elan Pharmaceuticals, Inc.; Eli Lilly and Company; EuroImmun; F. Hoffmann-La Roche Ltd and its affiliated company Genentech, Inc.; Fujirebio; GE Healthcare; IXICO Ltd.; Janssen Alzheimer Immunotherapy Research \& Development, LLC.; Johnson \& Johnson Pharmaceutical Research \& Development LLC.; Lumosity; Lundbeck; Merck \& Co., Inc.;

https://www.biorxiv. org/content/early/ 2018/02/19/267591

51,52 
bioRxiv preprint doi: https://doi.org/10.1101/303164; this version posted April 17, 2018. The copyright holder for this preprint (which was not certified by peer review) is the author/funder. All rights reserved. No reuse allowed without permission.

\section{Kaufmann et al., Genetics of brain age suggest an overlap with common brain disorders}

BETULA
CAMCAN
CIMH
CORR
DLBS
DS000030 (CNP)
DS000115
(CCNMD)
DS000119
DS000171
DS000202
DS000222
HCP

\section{Authors}

https://camcan-archive.mrccbu.cam.ac.uk/dataaccess/

Authors

http://fcon_1000.projects.nitrc.org/

http://fcon_1000.projects.nitrc.org/

https://openfmri.org/

https://openfmri.org/

https://openfmri.org/

https://openfmri.org/

https://openfmri.org/

https://openfmri.org/

https://www.humanconnectome.org/
HUNT

IXI

KASP https://www.ntnu.edu/hunt

http://brain-development.org/ixidataset/

Authors
Meso Scale Diagnostics, LLC.; NeuroRx Research; Neurotrack Technologies; Novartis Pharmaceuticals Corporation; Pfizer Inc.; Piramal Imaging; Servier; Takeda Pharmaceutical Company; and Transition Therapeutics. The Canadian Institutes of Health Research is providing funds to support ADNI clinical sites in Canada. Private sector contributions are facilitated by the Foundation for the National Institutes of Health (www.fnih.org). The grantee organization is the Northern California Institute for Research and Education, and the study is coordinated by the Alzheimer's Therapeutic Research Institute at the University of Southern California. ADNI data are disseminated by the Laboratory for Neuro Imaging at the University of Southern California.

Betula was supported by a Wallenberg Scholar Grant (KAW).

Data collection and sharing for this project was provided by the Cambridge Centre for Ageing and Neuroscience (CamCAN). CamCAN funding was provided by the UK Biotechnology and Biological Sciences Research Council (grant number BB/H008217/1), together with support from the UK Medical Research Council and University of Cambridge, UK.

CIMH was supported by the Deutsche Forschungsgesellschaft (DFG, projects ZI1253/3-1, ZI1253/3-2, KI 576/14-2, ME 1591/62) and the European Community's Seventh Framework Programme (FP7/2007-2013) grant agreement \#602450 (IMAGEMEND)

DS* data sets were obtained from the OpenfMRI database.

66,67 $\underline{D S 000030}$ work was supported by the Consortium for Neuropsychiatric Phenomics (NIH Roadmap for Medical Research grants UL1-DE019580, RL1MH083268, RL1MH083269, RL1DA024853, RL1MH083270, RL1LM009833, PL1MH083271, and PL1NS062410). DS000115 was supported through NIH Grants P50 MH071616 and R01 MH56584. DS000119 was supported by the National Institutes of Mental Health (NIMH RO1 MH067924). Enami Yasui provided assistance with data collection. DSO00171: Trisha Patrician and Natalie Stroupe assisted with screening of participants. Allan Schmitt and Franklin Hunsinger collected the MR data.

Data were provided [in part] by the Human Connectome Project, MGH-USC Consortium (Principal Investigators: Bruce R. Rosen, Arthur W. Toga and Van Wedeen; U01MH093765) funded by the NIH Blueprint Initiative for Neuroscience Research grant; the National Institutes of Health grant P41EB015896; and the Instrumentation Grants S10RR023043, 1S10RR023401, 1S10RR019307.

This study was supported by the Swedish Research Council (20062992, 2006-986, K2007-62X-15077-04-1, 2008-2167, K2008-62P20597-01-3. K2010-62X-15078-07-2, K2012-61X-15078-09-3, 2017-00949), the regional agreement on medical training and clinical research between Stockholm County Council and the Karolinska Institutet, the Knut and Alice Wallenberg Foundation, and the HUBIN project.

The HUNT Study is a collaboration between HUNT Research Centre, Faculty of Medicine and Health Sciences, Norwegian University of Science and Technology (NTNU), Nord-Trøndelag County Council, Central Norway Regional Health Authority, and the Norwegian Institute of Public Health. HUNT-MRI and the genetic analysis were funded by grants from the Liaison Committee between the Central Norway Regional Health Authority and NTNU to principal investigator Asta Håberg, and the Norwegian National Advisory Unit for functional MRI. We thank the HUNT MRI participants, MRI technicians and the Department of Diagnostic Imaging at Levanger Hospital, Professor Lars Jacob Stovner (NTNU) and the administrative staff at HUNT.

KaSP was supported by grants from the Swedish Medical Research Council (SE: 2009-7053; 2013-2838; SC: 523-2014-3467), the Council (SE: 2009-7053; 2013-2838; SC: 523-2014-3467), the
Swedish Brain Foundation, Ahlén-siftelsen, Svenska Läkaresällskapet, Petrus och Augusta Hedlunds Stiftelse, Torsten Söderbergs Stiftelse, the AstraZeneca-Karolinska Institutet Joint Research Program in Translational Science, Söderbergs Königska Stiftelse, Professor Bror Gadelius Minne, Knut och Alice Wallenbergs stiftelse, Stockholm County Council (ALF and PPG), Centre for Psychiatry Research, KID-funding from the Karolinska Institutet.

The study was funded by the South-Eastern Norway Regional 
bioRxiv preprint doi: https://doi.org/10.1101/303164; this version posted April 17, 2018. The copyright holder for this preprint (which was not certified by peer review) is the author/funder. All rights reserved. No reuse allowed without permission.

\section{Kaufmann et al., Genetics of brain age suggest an overlap with common brain disorders}

NCNG Authors

NIMAGE

Authors

NORCOG

Authors

OASIS
PING

PNC

RSI-MS

SALD

SCHIZCONNECT1 SCHIZCONNECT2 foundation.

The sample collection was supported by grants from the Bergen Research Foundation and the University of Bergen, the Dr Einar Martens Fund, the K.G. Jebsen Foundation, the Research Council of Norway, to SLH, VMS, AJL, and TE. The authors thank Dr. Eike Wehling for recruiting participants in Bergen, and Professor Jonn-Terje Geitung and Haraldplass Deaconess Hospital for access to the MRI facility. Additional support by RCN grants $177458 /$ V50 and $231286 / \mathrm{F} 20$.

This project was supported by grants from National Institutes of Health (grant R01MH62873 to SV Faraone) for initial sample recruitment, and from NWO Large Investment (grant 1750102007010 to JK Buitelaar), NWO Brain \& Cognition (grant 433-09-242 to JK Buitelaar), ZonMW Grant 60-60600-97-193, and grants from Radboud University Medical Center, University Medical Center Groningen, Accare, and VU University Amsterdam for subsequent assessment waves. NeuroIMAGE also receives funding from the European Community's Seventh Framework Programme (FP7/2007 - 2013) under grant agreements $n^{\circ} 602805$ (Aggressotype), $\mathrm{n}^{\circ} 278948$ (TACTICS), and $\mathrm{n}^{\circ} 602450$ (IMAGEMEND), and from the European Community's Horizon 2020 Programme (H2020/2014 - 2020) under grant agreements $n^{\circ}$ 643051 (MiND) and ${ }^{\circ} 667302$ (CoCA).

The Norwegian register of persons assessed for cognitive symptoms (NorCog) includes clinical, imaging and biological data from memory clinics in Norway (https://www.aldringoghelse.no/norkog/). The register is owned by Oslo University Hospital and administered by Norwegian National Advisory Unit on Ageing and Health.

The study was supported by grants P50 AG05681, P01 AG03991, R01 AG021910, P50 MH071616, U24 RR021382, R01 MH56584. Data used in the preparation of this article were obtained from the Pediatric Imaging, Neurocognition and Genetics (PING) Study database (http://ping.chd.ucsd.edu/). PING was launched in 2009 by the National Institute on Drug Abuse (NIDA) and the Eunice Kennedy Shriver National Institute Of Child Health \& Human Development (NICHD) as a 2-year project of the American Recovery and Reinvestment Act. The primary goal of PING has been to create a data resource of highly standardized and carefully curated magnetic resonance imaging (MRI) data, comprehensive genotyping data, and developmental and neuropsychological assessments for a large cohort of developing children aged 3 to 20 years. The scientific aim of the project is, by openly sharing these data, to amplify the power and productivity of investigations of healthy and disordered development in children, and to increase understanding of the origins of variation in neurobehavioral phenotypes. For up-to-date information, see http://ping.chd.ucsd.edu/."

Data collection and sharing for this project was funded by the Pediatric Imaging, Neurocognition and Genetics Study (PING) (National Institutes of Health Grant RC2DA029475). PING is funded by the National Institute on Drug Abuse and the Eunice Kennedy Shriver National Institute of Child Health \& Human Development. PING data are disseminated by the PING Coordinating Center at the Center for Human Development, University of California, San Diego.

Support for the collection of the data sets was provided by grant RC2MH089983 awarded to R. Gur and RC2MH089924 awarded to H. Hakonarson

Data collection in this MS cohort was supported by the SouthEastern Norway Regional Health Authority project 39569, Research Council of Norway grant 240102 and 240102, Oslo MS Society, Odd Fellow's Society for MS research.

Healthy controls were sampled from the TOP study (same scanner).

http://fcon_1000.projects.nitrc.org/

http://schizconnect.org/

Data used in preparation of this article were obtained from the SchizConnect (http://schizconnect.org) database. As such, the investigators within SchizConnect contributed to the design and implementation of SchizConnect and/or provided data but did not participate in analysis or writing of this report. Data collection and sharing for this project was funded by NIMH cooperative agreement 1U01 MH097435

SCHIZCONNECT1 comprised BrainGluSchi, COBRE and MCIC samples (COINS). SCHIZCONNECT2 comprised NUSDAST and NUNDA samples. Duplicate subjects in different sources were excluded.

The respective samples were supported by the following grants: BrainGluSchi: NIMH R01MH084898-01A1. COBRE: 5P20RR021938 /P20GM103472 from the NIH to Dr. Vince 
bioRxiv preprint doi: https://doi.org/10.1101/303164; this version posted April 17, 2018. The copyright holder for this preprint (which was not certified by peer review) is the author/funder. All rights reserved. No reuse allowed without permission.

Kaufmann et al., Genetics of brain age suggest an overlap with common brain disorders

\begin{tabular}{|c|c|c|c|}
\hline & & $\begin{array}{l}\text { Calhoun. } M C I C \text { : Department of Energy under Award Number DE- } \\
\text { FG02-08ER64581. NUSDAST: NIMH Grant 1R01 MH084803. } \\
\text { NUNDA: MH056584. }\end{array}$ & \\
\hline SCORE & Authors & $\begin{array}{l}\text { This work was supported by the Swiss National Science Foundation } \\
\text { (grant No. 119382) }\end{array}$ & 100,101 \\
\hline SLIM & http://fcon_1000.projects.nitrc.org/ & 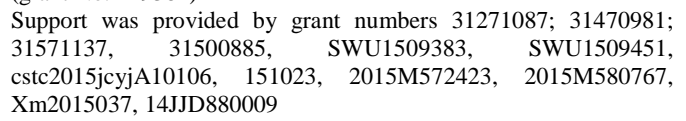 & 102,103 \\
\hline STROKEMRI/ MOT & Authors & $\begin{array}{l}\text { Supported by the Research Council of Norway ( } 249795,248238) \text {, } \\
\text { the South-Eastern Norway Regional Health Authority (2014097, } \\
2015044,2015073,2016083) \text {, and the Norwegian ExtraFoundation } \\
\text { for Health and Rehabilitation (2015/FO5146). }\end{array}$ & 104 \\
\hline TOP & Authors & $\begin{array}{l}\text { The work was funded by the Research Council of Norway (213837, } \\
223273,204966 / F 20,213694,229129,249795 / F 20,248778) \text {, the } \\
\text { South-Eastern Norway Regional Health Authority (2013-123, } \\
\text { 2014-097, 2015-073, \#2017-112) and Stiftelsen Kristian Gerhard } \\
\text { Jebsen. }\end{array}$ & $105-108$ \\
\hline UBA & Authors & $\begin{array}{l}\text { European Community's Seventh Framework Programme } \\
\text { (FP7/2007-2013) grant agreement \#602450 (IMAGEMEND) }\end{array}$ & 109 \\
\hline UKBB & https://www.ukbiobank.ac.uk/ & $\begin{array}{l}\text { This research has been conducted using the UK Biobank Resource } \\
\text { (access code 27412). All subjects with a primary or secondary ICD- } \\
10 \text { diagnosis with a mental or neurological disorder were excluded } \\
\text { prior to analysis and the remaining subjects included as healthy } \\
\text { controls. }\end{array}$ & 110 \\
\hline UNIBA & Authors & $\begin{array}{l}\text { This work was supported by a "Capitale Umano ad Alta } \\
\text { Qualificazione" grant by Fondazione Con Il Sud awarded to } \\
\text { Alessandro Bertolino and by a Hoffmann-La Roche Collaboration } \\
\text { Grant awarded to Giulio Pergola. This project has received funding } \\
\text { from the European Union Seventh Framework Programme for } \\
\text { research, technological development and demonstration under grant } \\
\text { agreement no. } 602450 \text { (IMAGEMEND). This paper reflects only } \\
\text { the author's views and the European Union is not liable for any use } \\
\text { that may be made of the information contained therein. }\end{array}$ & 111 \\
\hline
\end{tabular}

694 Suppl. Table 2: Summary of group size, age and sex for each cohort.

\begin{tabular}{|c|c|c|c|}
\hline Cohort & $\begin{array}{l}\text { Number of subjects per } \\
\text { group }\end{array}$ & $\begin{array}{l}\text { Age in years: mean } \pm \text { sd | } \\
\text { range (group) }\end{array}$ & Sex: $\mathbf{f} / \mathrm{m}$ \\
\hline ABIDE1 & $561(\mathrm{HC}) 517(\mathrm{ASD})$ & \begin{tabular}{l|l}
$17.1 \pm 7.8$ & $6.5-56.2(\mathrm{HC})$ \\
$17.2 \pm 8.5$ & $7.0-64.0(\mathrm{ASD})$
\end{tabular} & $161 / 917$ \\
\hline ABIDE2 & 498 (HC) 440 (ASD) & $\begin{array}{l}15.8 \pm 9.9 \mid 5.9-64.0(\mathrm{HC}) \\
15.4 \pm 9.6\end{array}$ & $212 / 726$ \\
\hline $\mathrm{ABM}$ & 73 (HC) 192 (MDD) & $\begin{array}{l}42.2 \pm 13.3 \mid 19.0-64.0(\mathrm{HC}) \\
39.3 \pm 13.4 \mid 18.0-71.0(\mathrm{MDD})\end{array}$ & $179 / 86$ \\
\hline AddNeuroMed & $\begin{array}{l}126(\mathrm{HC}) 141(\mathrm{MCI}) 148 \\
(\mathrm{DEM})\end{array}$ & \begin{tabular}{l|l}
$73.3 \pm 6.5$ & $53.0-87.8(\mathrm{HC})$ \\
$74.8 \pm 5.9$ & $56.8-90.1(\mathrm{MCI})$ \\
$76.0 \pm 6.5$ & $58.2-88.6(\mathrm{DEM})$
\end{tabular} & $237 / 178$ \\
\hline ADHD200 & 542 (HC) 320 (ADHD) & $\begin{array}{l}11.3 \pm 3.0 \\
10.6 \pm 2.5\end{array}$ & $322 / 540$ \\
\hline ADHDWUE & 55 (HC) 59 (ADHD) & $\begin{array}{l}40.0 \pm 12.1 \mid 24.0-61.0(\mathrm{HC}) \\
40.1 \pm 10.7 \mid 18.0-62.0(\mathrm{ADHD})\end{array}$ & $56 / 58$ \\
\hline ADNI1 & $\begin{array}{l}218(\mathrm{HC}) 386(\mathrm{MCI}) 187 \\
(\mathrm{DEM})\end{array}$ & \begin{tabular}{l|l}
$75.9 \pm 5.1$ & $59.9-89.6(\mathrm{HC})$ \\
$74.7 \pm 7.4$ & $54.4-89.3(\mathrm{MCI})$ \\
$75.2 \pm 7.5$ & $55.1-90.9(\mathrm{DEM})$
\end{tabular} & $334 / 457$ \\
\hline ADNI2 & $\begin{array}{l}268(\mathrm{HC}) 323(\mathrm{MCI}) 139 \\
(\mathrm{DEM})\end{array}$ & \begin{tabular}{l|l}
$72.9 \pm 6.0$ & $56.2-90.1(\mathrm{HC})$ \\
$71.7 \pm 7.3$ & $55.0-91.4(\mathrm{MCI})$ \\
$74.5 \pm 7.9$ & $55.6-90.3(\mathrm{DEM})$
\end{tabular} & $347 / 383$ \\
\hline BETULA & $347(\mathrm{HC})$ & $63.0 \pm 13.2 \mid 25.0-81.3(\mathrm{HC})$ & $181 / 166$ \\
\hline CAMCAN & $648(\mathrm{HC})$ & $54.2 \pm 18.6 \mid 18.0-88.0(\mathrm{HC})$ & $329 / 319$ \\
\hline CIMH & $43(\mathrm{HC}) 53(\mathrm{SZ})$ & $\begin{array}{l}30.6 \pm 11.5 \mid 18.0-60.0(\mathrm{HC}) \\
31.2 \pm 8.6 \mid 18.0-48.0(\mathrm{SZ})\end{array}$ & $31 / 65$ \\
\hline CNP & $\begin{array}{l}125(\mathrm{HC}) 41(\mathrm{ADHD}) 50 \\
(\mathrm{SZ}) 49(\mathrm{BD})\end{array}$ & $\begin{array}{l}31.5 \pm 8.8 \mid 21.0-50.0(\mathrm{HC}) \\
32.3 \pm 10.4 \mid 21.0-50.0(\mathrm{ADHD}) \\
36.5 \pm 8.9 \mid 22.0-49.0(\mathrm{SZ}) \\
35.3 \pm 9.0 \mid 21.0-50.0(\mathrm{BD})\end{array}$ & $112 / 153$ \\
\hline
\end{tabular}


Kaufmann et al., Genetics of brain age suggest an overlap with common brain disorders

\begin{tabular}{|c|c|c|c|}
\hline CoRR & $1360(\mathrm{HC})$ & $26.1 \pm 15.9 \mid 6.0-88.0(\mathrm{HC})$ & $694 / 666$ \\
\hline DLBS & $311(\mathrm{HC})$ & $54.3 \pm 20.0 \mid 20.6-89.1(\mathrm{HC})$ & $194 / 117$ \\
\hline ds000119 & $73(\mathrm{HC})$ & $16.2 \pm 4.7 \mid 8.1-26.8(\mathrm{HC})$ & $43 / 30$ \\
\hline ds000171 & 20 (HC) 19 (MDD) & $\begin{array}{l}29.4 \pm 11.3 \mid 18.0-59.0(\mathrm{HC}) \\
33.5 \pm 13.7 \mid 18.0-56.0(\mathrm{MDD})\end{array}$ & $22 / 17$ \\
\hline ds000202 & $94(\mathrm{HC})$ & $22.1 \pm 2.7 \mid 18.0-30.0(\mathrm{HC})$ & $94 / 0$ \\
\hline $\mathrm{ds} 000222$ & $79(\mathrm{HC})$ & $44.4 \pm 20.1 \mid 21.0-73.0(\mathrm{HC})$ & $41 / 38$ \\
\hline $\mathrm{HCP}$ & $1113(\mathrm{HC})$ & $28.8 \pm 3.7 \mid 22.0-37.0(\mathrm{HC})$ & $606 / 507$ \\
\hline HUBIN & $102(\mathrm{HC}) 94(\mathrm{SZ})$ & $\begin{array}{l}42.0 \pm 8.8 \mid 19.4-56.2(\mathrm{HC}) \\
41.7 \pm 7.6 \\
24.9-56.3(\mathrm{SZ})\end{array}$ & $57 / 139$ \\
\hline HUNT & $909(\mathrm{HC})$ & $58.8 \pm 4.2 \mid 50.5-66.8(\mathrm{HC})$ & $477 / 432$ \\
\hline IXI & $562(\mathrm{HC})$ & $48.6 \pm 16.5$ | 20.0-86.3 (HC) & $312 / 250$ \\
\hline KASP & $\begin{array}{l}45 \text { (HC) } 37 \text { (SZ) } 27 \\
\text { (PSYMIX) }\end{array}$ & \begin{tabular}{l|l}
$25.7 \pm 5.2$ & $19.0-43.0(\mathrm{HC})$ \\
$28.5 \pm 7.5$ & $18.0-50.0(\mathrm{SZ})$ \\
$29.1 \pm 8.3$ & $18.0-48.0$ (PSYMIX)
\end{tabular} & $43 / 66$ \\
\hline MALTOSLO & 44 (HC) 44 (BD) & \begin{tabular}{l|l|l}
$31.2 \pm 9.1$ & $20.0-50.0(\mathrm{HC})$ \\
$34.4 \pm 7.3$ & $18.0-48.0(\mathrm{BD})$
\end{tabular} & $58 / 30$ \\
\hline MOT & $57(\mathrm{HC})$ & $25.3 \pm 3.9 \mid 21.0-36.0(\mathrm{HC})$ & $33 / 24$ \\
\hline NCNG & $465(\mathrm{HC})$ & $53.3 \pm 16.5 \mid 19.4-82.3(\mathrm{HC})$ & $310 / 155$ \\
\hline NIMAGE & 372 (HC) 331 (ADHD) & \begin{tabular}{l|l}
$16.9 \pm 3.8$ & $7.7-28.6(\mathrm{HC})$ \\
$16.8 \pm 3.6$ & $7.4-27.5(\mathrm{ADHD})$
\end{tabular} & $310 / 393$ \\
\hline NORCOG & $\begin{array}{l}312(\mathrm{HC}) 142(\mathrm{MCI}) 186 \\
(\mathrm{DEM})\end{array}$ & $\begin{array}{l}63.3 \pm 10.9 \mid 40.0-95.0(\mathrm{HC}) \\
65.0 \pm 10.2 \mid 38.1-85.2(\mathrm{MCI}) \\
71.5 \pm 8.1 \mid 52.9-90.7(\mathrm{DEM})\end{array}$ & $351 / 289$ \\
\hline OASIS & 315 (HC) 99 (DEM) & $\begin{array}{l}45.0 \pm 23.9 \mid 18.0-94.0(\mathrm{HC}) \\
76.9 \pm 7.1 \mid 62.0-96.0(\mathrm{DEM})\end{array}$ & $254 / 160$ \\
\hline PING & $1162(\mathrm{HC})$ & $12.0 \pm 5.1 \mid 3.0-21.0(\mathrm{HC})$ & $556 / 606$ \\
\hline PNC & $1466(\mathrm{HC})$ & $15.1 \pm 3.6 \mid 8.2-23.2(\mathrm{HC})$ & $775 / 691$ \\
\hline RSI-MS & $\begin{array}{l}254 \text { (MS), HC were } \\
\text { sampled from TOP } \\
\text { (same scanner) }\end{array}$ & $41.0 \pm 10.0 \mid 19.0-68.3(\mathrm{MS})$ & $196 / 58$ \\
\hline SALD & $493(\mathrm{HC})$ & 45.2 $217.4 \mid 19.0-80.0(\mathrm{HC})$ & $307 / 186$ \\
\hline SCHIZCONNECT1 & 230 (HC) 225 (SZ) & \begin{tabular}{l|l}
$35.2 \pm 12.2$ & $18.0-65.0(\mathrm{HC})$ \\
$36.3 \pm 13.0$ & $18.0-66.0(\mathrm{SZ})$
\end{tabular} & $117 / 338$ \\
\hline SCHIZCONNECT2 & 145 (HC) 158 (SZ) & \begin{tabular}{l|l}
$32.3 \pm 13.1$ & $14.0-68.1(\mathrm{HC})$ \\
$34.4 \pm 11.7$ & $19.0-62.4(\mathrm{SZ})$
\end{tabular} & $120 / 183$ \\
\hline SCORE & $\begin{array}{l}44 \text { (HC) } 80 \text { (SZRISK) } 78 \\
\text { (PSYMIX) }\end{array}$ & \begin{tabular}{l|l}
$25.5 \pm 4.3$ & $19.0-39.0$ (HC) \\
$24.3 \pm 5.2$ & $18.0-42.0$ (SZRISK) \\
$26.9 \pm 6.7$ & $18.0-42.0$ (PSYMIX)
\end{tabular} & $69 / 133$ \\
\hline SLIM & $556(\mathrm{HC})$ & $20.1 \pm 1.2 \mid 17.0-27.0(\mathrm{HC})$ & $316 / 240$ \\
\hline STROKEMRI & $61(\mathrm{HC})$ & $27.7 \pm 5.6 \mid 18.0-40.0(\mathrm{HC})$ & $36 / 25$ \\
\hline TOP & $\begin{array}{l}958 \text { (HC) } 18 \text { (ASD) } 18 \\
\text { (SZRISK) } 444 \text { (SZ) } 189 \\
\text { (PSYMIX) } 352 \text { (BD) }\end{array}$ & 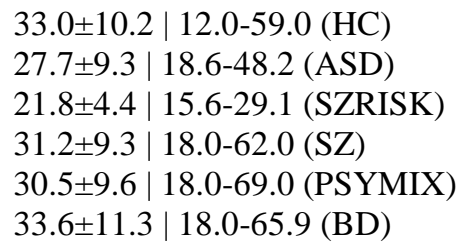 & $913 / 1066$ \\
\hline UBA & $1099(\mathrm{HC})$ & $22.5 \pm 3.4 \mid 18.0-35.0(\mathrm{HC})$ & $714 / 385$ \\
\hline UKBB & $14581(\mathrm{HC})$ & $55.7 \pm 7.5 \mid 40.2-70.2(\mathrm{HC})$ & $7573 / 7008$ \\
\hline UNIBA & 435 (HC) 84 (SZ) & \begin{tabular}{l|l|}
$26.7 \pm 7.7$ & $18.0-63.0(\mathrm{HC})$ \\
$34.3 \pm 7.7$ & $19.0-58.0(\mathrm{SZ})$
\end{tabular} & $246 / 273$ \\
\hline
\end{tabular}


Kaufmann et al., Genetics of brain age suggest an overlap with common brain disorders

695 Suppl. Table 3: Significant loci from conjunctional FDR analysis reflecting overlap between brain

696 age gaps and the respective disorders. The gene column reflects the gene closest to the significant SNP

697 as identified via the Ensembl Variable Effect Predictor ${ }^{47}$, unless the closest gene is more than 5000 bp

698 away in which case no annotation is provided.

\begin{tabular}{|c|c|c|c|c|c|c|}
\hline $\begin{array}{l}\text { Brain region, } \\
\text { group }\end{array}$ & Lead SNP & $\begin{array}{l}\text { Chr. } \\
\text { region }\end{array}$ & $\begin{array}{l}\text { Chr. } \\
\text { position }\end{array}$ & $\begin{array}{l}\text { Conj. } \\
\text { FDR }\end{array}$ & Consequence & Gene \\
\hline occipital, SZ & rs354232 & $2 \mathrm{p} 16.2$ & 54916910 & 0.042 & Intergenic variant & - \\
\hline occipital, SZ & rs994261 & $2 \mathrm{q} 33.1$ & 199908378 & 0.033 & $\begin{array}{l}\text { Intron variant, } \\
\text { non-coding } \\
\text { transcript variant }\end{array}$ & AC018717.1 \\
\hline frontal, SZ & rs10803691 & $2 \mathrm{p} 25.2$ & 5449582 & 0.047 & $\begin{array}{l}\text { Downstream gene } \\
\text { variant }\end{array}$ & AC073143.1 \\
\hline frontal, SZ & rs12820906 & $12 q 24.31$ & 123493123 & 0.014 & Intron variant & PITPNM2 \\
\hline frontal, SZ & rs4981695 & $14 q 12$ & 29700996 & 0.028 & Intergenic variant & - \\
\hline frontal, SZ & rs221923 & $14 \mathrm{q} 24.2$ & 71584473 & 0.035 & $\begin{array}{l}\text { Downstream gene } \\
\text { variant }\end{array}$ & PCNX \\
\hline temporal, SZ & rs11892879 & $2 \mathrm{q} 24.1$ & 156114425 & 0.048 & Intergenic variant & - \\
\hline temporal, SZ & rs4689287 & $4 \mathrm{p} 16.2$ & 5990110 & 0.049 & Missense variant & C4orf50 \\
\hline temporal, SZ & rs2764264 & $6 q 21$ & 108934461 & 0.0065 & Intron variant & FOXO3 \\
\hline parietal, SZ & rs9379851 & $6 \mathrm{p} 22.2$ & 26354780 & 0.0061 & $\begin{array}{l}\text { Upstream gene } \\
\text { variant }\end{array}$ & RNU6-1259P \\
\hline cingulate, SZ & rs6704641 & $2 q 33.1$ & 200164252 & 0.031 & Intron variant & SATB2 \\
\hline cingulate, $\mathrm{SZ}$ & rs7729320 & $5 \mathrm{p} 13.1$ & 38725149 & 0.04 & $\begin{array}{l}\text { Upstream gene } \\
\text { variant }\end{array}$ & RP11-122C5.1 \\
\hline insula, SZ & rs10460398 & $2 q 33.1$ & 200214742 & 0.0061 & Intron variant & SATB2 \\
\hline subcortical, SZ & rs673253 & $1 \mathrm{p} 34.2$ & 44062154 & 0.045 & Intron variant & PTPRF \\
\hline subcortical, SZ & rs13107325 & $4 q 24$ & 103188709 & 0.00026 & Missense variant & SLC39A8 \\
\hline frontal, BD & rs9489193 & $6 q 22.1$ & 117769728 & 0.039 & $\begin{array}{l}\text { Downstream gene } \\
\text { variant }\end{array}$ & RP1-92C8.3 \\
\hline frontal, BD & rs 10160070 & $10 \mathrm{q} 21.1$ & 54201572 & 0.046 & Intergenic variant & - \\
\hline frontal, BD & rs1884431 & $20 \mathrm{q} 11.22$ & 33338585 & 0.05 & Intron variant & NCOA6 \\
\hline cingulate, BD & rs 174583 & $11 \mathrm{q} 12.2$ & 61609750 & 0.016 & Intron variant & FADS2 \\
\hline cingulate, $\mathrm{BD}$ & rs1034589 & $22 q 12.2$ & 31579233 & 0.013 & Intron variant & RNF185 \\
\hline insula, BD & rs2273684 & $20 q 11.22$ & 33529766 & 0.035 & Intron variant & GSS \\
\hline full brain, MS & rs4465231 & $1 \mathrm{p} 36.22$ & 9347278 & 0.018 & Intergenic variant & - \\
\hline full brain, MS & rs 12146713 & $12 \mathrm{q} 23.3$ & 106476805 & 0.02 & Intron variant & NUAK1 \\
\hline frontal, MS & rs11164855 & $1 \mathrm{p} 22.1$ & 93466960 & 0.032 & Intergenic variant & - \\
\hline frontal, MS & rs940904 & $12 \mathrm{q} 24.31$ & 123491572 & 0.032 & Intron variant & PITPNM2 \\
\hline temporal, MS & rs 10484566 & $6 \mathrm{p} 21.32$ & 32835258 & 0.045 & $\begin{array}{l}\text { Regulatory region } \\
\text { variant }\end{array}$ & - \\
\hline subcortical, MS & rs6482190 & $10 \mathrm{p} 12.31$ & 22037809 & 0.049 & Intergenic variant & - \\
\hline subcortical, MS & rs12146713 & $12 q 23.3$ & 106476805 & 0.026 & Intron variant & NUAK1 \\
\hline temporal, $\mathrm{AD}$ & rs6485758 & $11 \mathrm{p} 11.2$ & 47530024 & 0.046 & Intron variant & CELF1 \\
\hline
\end{tabular}

